\title{
A Quantitative Model for Disruption Mitigation in a Supply Chain
}

\author{
Sanjoy Kumar Paul, Ruhul Sarker and Daryl Essam
}

\begin{abstract}
In this paper, a three-stage supply chain network, with multiple manufacturing plants, distribution centers and retailers, is considered. For this supply chain system we develop three different approaches, (i) an ideal plan for an infinite planning horizon and an updated plan if there are any changes in the data, (ii) a predictive mitigation planning approach for managing predictive demand changes, which can be predicted in advance by using an appropriate tool, and (iii) a reactive mitigation plan, on a real-time basis, for managing sudden production disruptions, which cannot be predicted in advance. In predictive mitigation planning, we develop a fuzzy inference system (FIS) tool to predict the changes in future demand over the base forecast and the supply chain plan is revised accordingly well in advance. In reactive mitigation planning, we formulate a quantitative model for revising production and distribution plans, over a finite future planning period, while minimizing the total supply chain cost. We also consider a series of sudden disruptions, where a new disruption may or may not affect the recovery plans of earlier disruptions and which consequently require plans to be revised after the occurrence of each disruption on a real-time basis. An efficient heuristic, capable of dealing with sudden production disruptions on a realtime basis, is developed. We compare the heuristic results with those obtained from the LINGO optimization software for a good number of randomly generated test problems. Also, some numerical examples are presented to explain both the usefulness and advantages of the proposed approaches.
\end{abstract}

Keywords: supply chain; mitigation; production disruption; quantitative model; heuristic.

\section{INTRODUCTION}

A supply chain is a network that receives inputs or raw materials from suppliers, produces final products at its manufacturing facilities and delivers those products to customers through a distribution network. Every manufacturing and service industry is part of a supply chain network which can have multiple manufacturing plants, multiple distribution centers (DCs) and multiple retailers. There are numerous industries, such as the pharmaceutical, textile and manufacturing, that supply, produce and distribute products using a supply chain network. 
Depending on the number of entities in each tier of a network, it can be very complex, and in a real-life one, any information can be changed at any time. Therefore, an ideal plan should be updated to incorporate changes in order to generate a better plan. Although some changes in data may be known well in advance, others may not, but can instead be detected using appropriate prediction tools. Such predictions will help to generate a better supply chain plan than the one designed for ideal conditions. In real-world supply chain system, the plan should be revised if there are any changes in data and/or if any future changes that can be predicted in advance. In this paper, we aim to develop both updated and predictive mitigation plans in a three-stage supply chain system to incorporate any known changes in data and predicted changes in demand respectively.

Supply chain entities can also face many sudden uncontrollable problems, which cannot be predicted in advance, such as a production disruption in a manufacturing plant, which can be defined as any form of interruption in the manufacturing system, including a material shortage, machine breakdown, or any other form of accidental or man-made disturbance (Paul et al., 2016a). Disruption management is an important research topic in supply chain, as is obvious in the following examples. A recent study conducted in 2015 by the Business Continuity Institute (Supply Chain Resilience Report, 2015) reports that, although the awareness of supply chain risks is increasing, many companies remain exposed to high levels of risk. It states that $74 \%$ of survey respondents from 426 organizations had experienced at least one disruption in their supply chain, with 6-20 disruptions per year for $50 \%$ of the companies, and the financial losses varied from 50 thousand to 500 million euros. More than $23 \%$ of the companies reported that the loss due to a disruption is at least one million euros. Supply chain disruptions not only cause financial loss but can also damage a company's brand or reputation as a result of third-party failures. It has been reported that $27 \%$ companies have suffered damage to their reputations, 58\% lost productivity and 38\% lost revenue. According to Sodhi and Chopra (2004), a disruption at the Royal Phillips Electronics plant in New Mexico on March 17, 2000 was caused by lightning strikes which led to a massive surge in the surrounding electrical grid, and later, a resultant fire damaged millions of microchips. Nokia Corporation and Ericsson were two major customers of the Phillips plant. To obtain a backup supply, immediately after this fire disaster, Nokia took proactive measures by redesigning its products and switching its chip orders to other Phillips plants. In contrast, as Ericsson employed a single sourcing policy and a slow disruption recovery plan, its production was disrupted for months, which caused 400 million US dollar in lost sales. From 
the above two examples, it is clear that supply, production and distribution systems can be unbalanced due to a disruption, and consequently, organizations can face enormous financial losses as well as loss of customer goodwill. Though disruption mitigation is an important research topic in supply chain, in the literature a very few papers developed quantitative reactive mitigation approaches in production-inventory system but no study extended the concept for multi-stage supply chain system. Therefore, it is essential to extend the concept of disruption recovery to develop an appropriate reactive mitigation model for a supply chain system for minimizing the effect of a sudden disruption. To fulfil this gap in the literature, this paper also aims to develop a new quantitative reactive mitigation model for managing both single and a series of sudden production disruptions in a three-stage supply chain system.

Production-inventory system is considered as a sub-set of a practical supply chain system. For this reason, most researchers concentrated their study in production-inventory system and some others extended their work to supply chain environments. At first, the researchers focused on developing models under ideal conditions, for example, based on distribution systems with a single product, single warehouse and multiple retailers (Petrovic et al., 2008), a single manufacturer and single retailer, with the demand and manufacturing cost fuzzy variables (Zhou et al., 2008), a single period and two-stage supply chain coordination problem (Xu and Zhai, 2010) and a three-stage system consisting of supplier, manufacturer and retailer producing a combination of perfect and/or defective items (Sana, 2011; 2012). Recently, Pal et al. (2012) developed an inventory model for multiple items production with multiple suppliers, one manufacturer and multiple retailers with deterministic demand. A few more studies on supply chain models under ideal conditions, can be found in Purnomo et al. (2012), Reza Nasiri et al. (2014), Paul et al. (2014), and Heydari (2014),

The above studies, and many others, used perfect supply chain environments. In real-life supply chain systems, there may be some known changes in data, such as changes in cost and capacity. Although some changes in data may be known well in advance, others may not, but rather need to be predicted using an appropriate tool. Most of the papers considered traditional forecasting techniques to predict supply change events (Syntetos et al., 2016). But an appropriate prediction tool, which can be used to predict any future changes over the base forecast, can be helpful for developing an efficient quantitative predictive mitigation plan. In this paper, one of our objectives is to develop an appropriate prediction tool to predict the 
changes in demand and to develop a quantitative predictive mitigation plan based on the prediction.

Over the last decade, developing reactive mitigation plan for managing sudden disruption has become an important research topic in supply chain system. If a system is disrupted for a given period of time (known as a disruption period/duration), it is necessary to revise, after a disruption, the production schedule (known as a recovery plan) for some periods in the future (known as a recovery time window) until the system returns to its normal schedule (Hishamuddin et al., 2012). In modelling, the recovery time window can be either user specified or a variable that must be determined. In recent years, Fahimnia et al. (2015), Snyder et al. (2016), Paul et al. (2016a) provided an extensive review of supply chain risk and disruption management models. However, in this paper, we review mainly recent researches on sudden disruption recovery models in production-inventory and supply chain systems.

Firstly, we discuss the disruption recovery models in production-inventory systems. Xia et al. (2004) proposed a general disruption management approach for a two-stage production and inventory control system that incorporates a penalty cost for deviations of the new plan from the original. They introduced the concept of a disruption recovery time window that was considered in most recent models. Eisenstein (2005) introduced a flexible dynamic produceup-to policy that is able to respond to a disruption by adjusting the amount of idle time during recovery and re-establishes the target idle time as a schedule is recovered. A production disruption recovery model, for a single disruption within a single-stage single-item production system, for obtaining a recovery plan within a user-defined time window was developed by Hishamuddin et al. (2012), which was basically an extension of the model of Xia et al. (2004). This study considered back orders and lost sales options as recovery strategies. This concept was further extended to develop a real-time disruption recovery model for managing both single and multiple disruptions in a single-stage productioninventory system (Paul et al., 2015a), a two-stage imperfect production-inventory system (Paul et al., 2014a), and a three-stage mixed production environment (Paul et al., 2015b) . In the same direction of research, an approach for managing demand fluctuation on a real-time basis in a supplier-retailer coordinated system was developed by Paul et al. (2014b). In the same research area, some other recovery models for deterministic demand in a productioninventory system can be found in Gallego (1994), Qi et al. (2004), Tang and Lee (2005), Yang et al. (2005), Paul et al. (2013) and Paul et al. (2014c). 
In the supply chain context, the delivery disruptions have been studied by a few researchers. Giunipero and Eltantawy (2004) discussed a transportation disruption in general in their study, but did not specify strategies for facing it. Wilson (2007) investigated the effects of a transportation disruption on the performance of a supply chain by using a system dynamics simulation in a 5-echelon supply chain system. Unnikrishnan and Figliozzi (2011) formulated a mathematical model for a new type of freight network assignment problem in a dynamic environment in the presence of probable network disruptions or significant delays. Recently, Hishamuddin et al. (2013) developed a transportation disruption recovery model in a twostage, single supplier and single retailer, supply chain system. Recently, the recovery modelling concept was further extended for managing a supply disruption in a two-stage supply chain consisting of a single supplier and single retailer (Hishamuddin et al., 2014) and for managing both single and multiple sudden supply disruptions in a three-stage supply chain with multiple suppliers and retailers (Paul et al., 2016b). Over the last few years, some other supply disruption management approaches have been studied by Li et al. (2004), Tolmin (2006), Craighead et al. (2007), Chopra et al. (2007), Wu et al. (2007), Ross et al. (2008), Mohebbi and Hao (2008), Qi et al. (2009), and Hult et al. (2010).

There are several gaps in the literature. It is clear that most researchers focused on supply chain coordination and optimization problems under ideal conditions, although a number of studies developed recovery and reactive mitigation models after the occurrence of a sudden disruption. However, no study has been found that predicts the possible changes in future demand that is used as input to the mitigation planning model in supply chain environment. Moreover, most past studies focused mainly on a single disruption in production and a very few focused on a series of disruptions on a real-time basis, but they are again for a single supplier and single retailer, which limits their applicability in real-life situations (Paul et al., 2016a). Interestingly, no study has been found which developed a quantitative recovery for managing sudden production disruption in a supply chain with multiple entities in each stage of the system.

In this paper, firstly, we have formulated a model to generate an ideal supply chain plan. If any variation in data in any period is observed, this plan will be updated for a finite period on a rolling horizon basis with the new data. In real-life situations, some changes may not be known in advance, but can be predicted using an appropriate prediction tool. Therefore, in this paper, a predictive mitigation planning approach is developed, with the predicted data used to generate a revised plan in advance on a rolling planning horizon basis. A fuzzy 
inference system (FIS) based tool is used to predict future changes in demand, and then such predicted demand data are used to develop a revised plan in advance, which is said to be a predictive mitigation plan. Finally, we have developed a disruption recovery (reactive mitigation) model for a supply chain network consisting of multiple manufacturing plants, DCs and retailers. We consider disruptions due to technical and internal problems, which take place more frequently (repetitive type) and are for short durations. In real-life, a system can face a series of production disruptions (known as multiple disruptions), one after another, at any plant. If a new disruption occurs at any plan during the revised planning window of a previous production disruption, known as a dependent disruption, the production and distribution plan must be revised again while considering the effects of both disruptions. Therefore, this can be a continuous process that must be dealt with on a real-time basis. A real-time disruption management scenario in a three-stage supply chain network, where the disruptions are not known a priori, is considered in this study. This means that the current plan is revised immediately after a disruption occurs, as this disruption is impossible to predict. For experimentation, as we assume that any disruption event is random, we generate disruption scenarios using a uniformly random distribution (Paul et al., 2015b) to determine characteristics such as the disruptions' start times and durations. However, it is possible to generate disruption scenarios by using other probability distributions. To achieve this objective, we develop a new mathematical and heuristic approach for obtaining a recovery plan after the occurrence of a single disruption or a series of disruptions on a real-time basis. The results for both the predictive and reactive mitigation approaches are discussed and some numerical examples are presented to demonstrate their usefulness.

The main contributions of this paper can be summarized as follows.

i. Developing an updated supply chain plan for a finite period on a rolling horizon basis to incorporate any changes in data.

ii. Developing a predictive mitigation planning approach for obtaining a better supply chain plan. A fuzzy inference system based tool is designed to predict any changes in base forecasted demand, based on information about fluctuation, unexpected incident and natural incident, and the supply chain plan is accordingly revised well in advance.

iii. Developing a mathematical model for managing sudden production disruptions which cannot be predicted in advance. The supply chain plan is revised, after the 
occurrence of a disruption, for a finite period into the future, on a real-time basis, to minimize loss due to the disruption.

iv. Developing a new heuristic for generating recovery plan for the sudden production disruption problem in iii. The heuristic results are compared with those from another established solution technique for a good number of randomly generated test problems.

v. Extending the heuristic to deal with multiple disruptions, one after another as a series, on a real-time basis. This heuristic is capable of determining a recovery plan, after the occurrence of each disruption, for as long as disruptions take place in the system.

The paper is organized as follows. In Section 2, we describe the problem, along with the notations and assumptions used in this study. In Sections 3 and 4 respectively, we present the model formulation and solution approach. In Section 5, we discuss the experimentation and analysis of results. Applicability of the model and value in practice are presented in Section 6. Finally, we provide a summary of, and future guidelines for, our research in Section 7.

\section{PROBLEM DESCRIPTION}

Firstly, this paper develops a supply chain model under ideal conditions for an infinite planning horizon. Basically, this ideal plan is used to determine the cycle length, which we consider the length of a period required for planning and analysis. A three-tier supply chain network with multiple entities in each tier (such as manufacturing plants, DCs and retailers) is considered, as presented in Figure 1. In the ideal system, products are produced in the manufacturing plants and then are moved to DCs, and finally, distributed to retailers from the DCs according to retailers' demands. The total supply chain cost is minimized to obtain the ideal production and distribution plan. The ideal system is formulated mathematically as a constrained programming problem, where the objective is to minimize the total supply chain cost, subject to capacity, distribution and demand constraints. The decision variables are the production quantity in each plant $i$, and the quantities transported from plant $i$ to DC $j$, and from $\mathrm{DC} j$ to retailer $k$. 


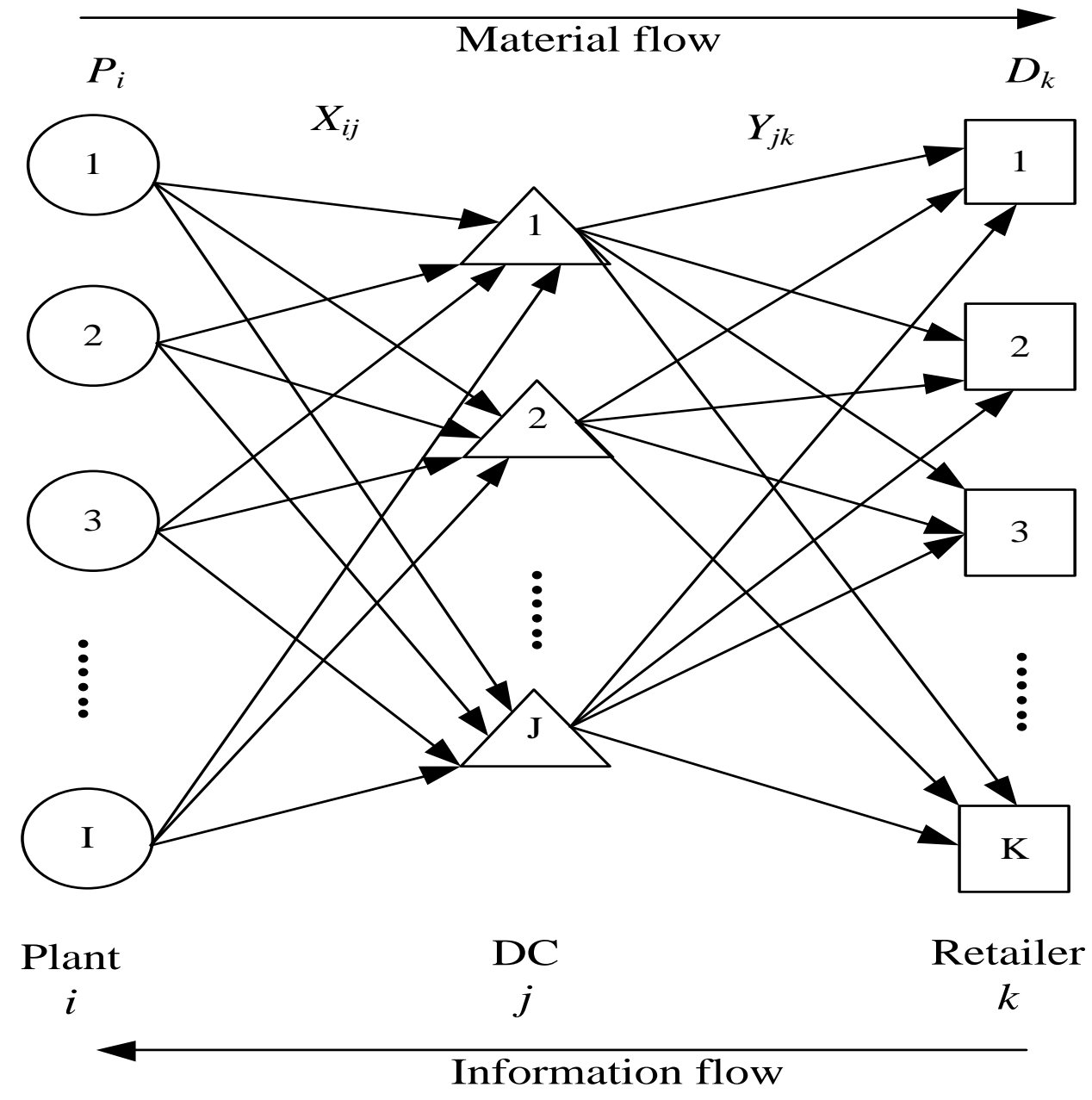

Figure 1: Ideal supply chain network for infinite planning horizon

In a real-life situation, a supply chain's input data may vary at any time due to, for example, changes in demand, cost, production capacity and amount of raw materials. If there is any variation in any period, the ideal plan must be updated with the new known information. In fact, the model is run on a rolling horizon basis to incorporate changes in the data and is known as an updated plan.

Some changes in the data may be known well in advance, as discussed above. However, others may not be, but can be predicted using appropriate prediction tools. Such predictions will help to generate a better plan than the one developed under ideal conditions. In this paper, the predicted data are used with the rolling horizon planning model to generate the revised plan, which is known as a predictive mitigation plan, and its methodology is illustrated in Figure 2. We use historical data to calculate the base forecast which we update by predicting some future event, such as a demand fluctuation, unexpected incident and natural incident, with examples presented in Table 1. We use qualitative information of the presence (Yes or 
No) of future events, and based on such information, we develop a rule and logic-based fuzzy inference system (FIS) to quantify the value of a qualitatively predicted event, with its working principle and different components illustrated in Figure 3 (Paul, 2015). A brief description of these components is as follows.

\section{i. $\quad$ FIS Editor}

In this editor, input and output variables are designed. The number of input and output variables can be edited.

\section{ii. Membership Function Editor}

In this editor, membership functions are designed for input and output linguistic variables. There are few types of membership functions such as Triangular, Gaussian, and Trapezoidal etc. The types and number of membership functions can be edited.

\section{iii. Rule Editor}

In this editor, pertinent rules are designed to relate the input linguistic variables to output. The numbers of rules are depended on the specific problem and number of input and outputs. These rules can be edited in this editor.

\section{iv. Rule Viewer}

In this viewer, decision maker can give input the value of multiple input variables and obtain the value of output of corresponding input.

\section{v. Surface Viewer}

In this viewer, the graphical relationship between input and output variables can be perceived. Based on the developed membership functions and pertinent rules, these relationships are obtained.

As we have to predict a change of demand based on three different input events, a FIS can be a useful tool, because FIS is an efficient tool for multi-criteria decision making (Paul, 2015). So, in this paper, the changes over the base forecast of demand are predicted by using a FIS tool, based on the qualitative information of future events, such as a demand fluctuation (Paul et al., 2014b), an unexpected incident (Sodhi and Chopra, 2004), or a natural incident (Cavallo et al., 2014). If there is no future event, we use the base forecast for a prediction on which we base our revision of the supply chain plan on a rolling horizon. A detailed description and step-by-step methodology of FIS with an application can be found in Ahmed et al. (2013), and Hasan et al. (2014).

We use the following steps to predict the changes in demand. 
Step 1: Determine input for the changes in demand and deploy in FIS editor.

Step 2: Develop the linguistic variables and membership functions for input and output.

Step 3: Develop the membership functions for linguistic variables by using membership function editor.

Step 4: Develop pertinent rules to relate input and output by using rule editor.

Step 5: Determine the changes in demand by providing information of input values in rule viewer.

The main advantages of using FIS tool, over the traditional prediction tools, are as follows.

i. It has intelligent capability to predict the output based on available input information (Paul, 2015).

ii. It is capable to handle multiple-criteria (Paul, 2015; Paul and Azeem, 2010).

iii. The rules are easy to write, and as many rules as necessary can be supplied to describe the system adequately (Ahmed et al., 2013).

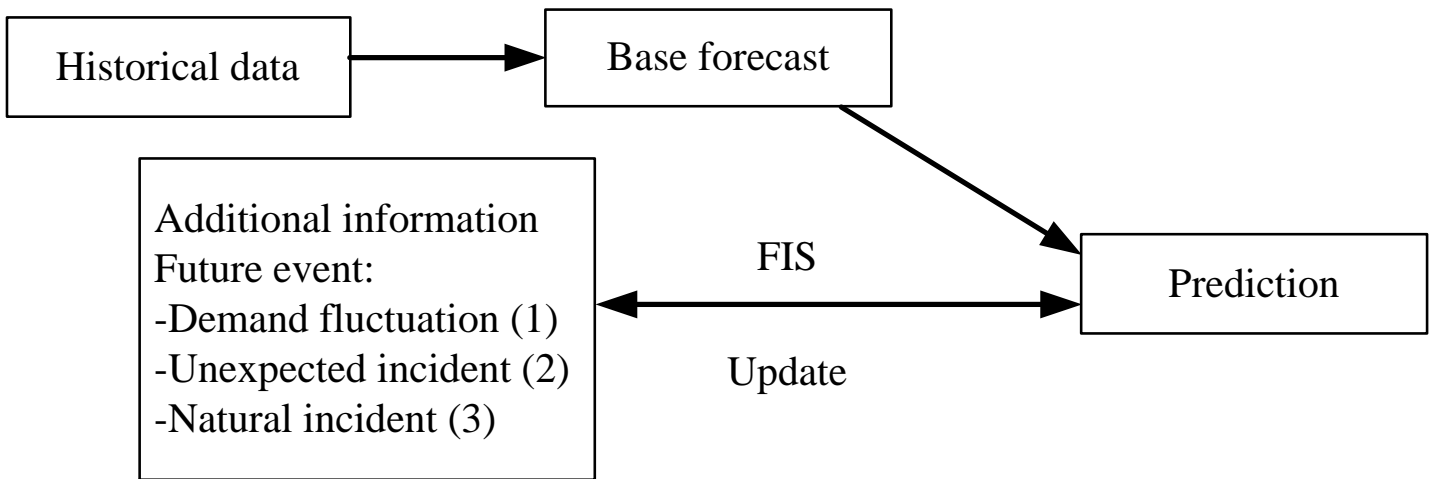

Figure 2: Process of prediction for predictive mitigation

Table 1: Examples of future events ( $\mathrm{Y}$-yes and $\mathrm{N}-\mathrm{no}$ )

\begin{tabular}{|c|c|c|c|c|}
\hline \multirow{2}{*}{ Event } & \multicolumn{4}{|c|}{ Predicted period } \\
\cline { 2 - 5 } & 1 & 2 & $\cdots \cdots$ & $\mathrm{F}$ \\
\hline Demand fluctuation & $\mathrm{N}$ & $\mathrm{N}$ & $\cdots \cdots$ & $\mathrm{Y}$ \\
\hline Unexpected incident & $\mathrm{N}$ & $\mathrm{Y}$ & $\cdots \cdots$ & $\mathrm{N}$ \\
\hline Natural incident & $\mathrm{Y}$ & $\mathrm{N}$ & $\cdots \cdots$ & $\mathrm{N}$ \\
\hline
\end{tabular}




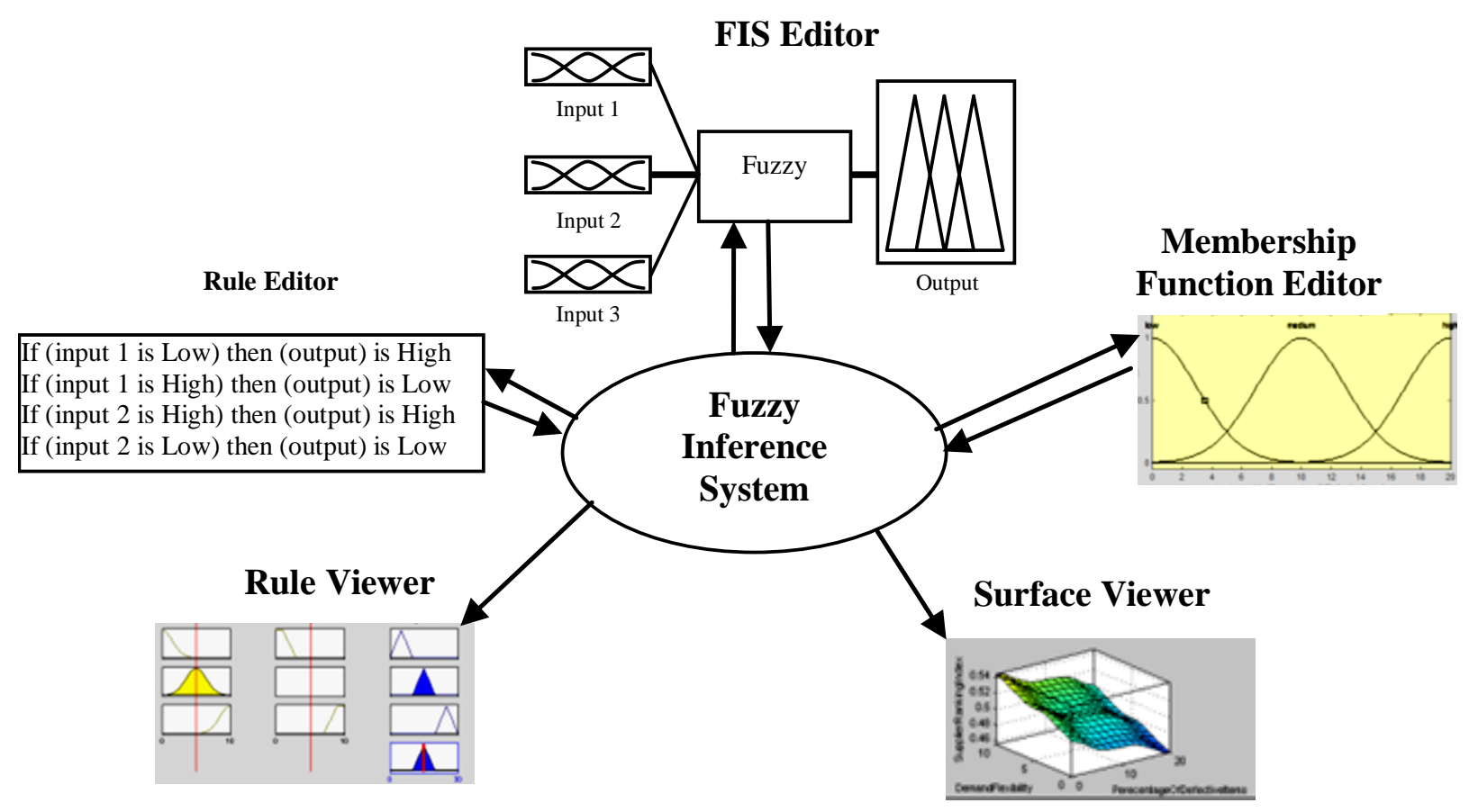

Figure 3: Rule and logic-based FIS (Paul, 2015)

Finally, this paper develops a recovery plan for managing sudden production disruptions, which is actually a reactive mitigation plan. In real-life situations, any supply chain can face a sudden disruption at any time. After such an occurrence, the production and distribution plan must be revised for a finite period in the future, so that losses can be minimized and the system returns to its ideal plan as quickly as possible.

A production disruption is a familiar event in any manufacturing environment. If there is a sudden disruption at any plant, as that plant will be inoperable for a certain period of time, there will be a loss of production quantity. Afterwards, the main objective is to minimize that loss by revising the production and distribution plan for a finite period in the future, with the revision mechanism presented in Figure 4. After the occurrence of a sudden disruption with a duration of $T_{d n}$, the supply chain plan is revised for a future finite planning period (e.g., for the next $M$ periods), which is known as a recovery window.

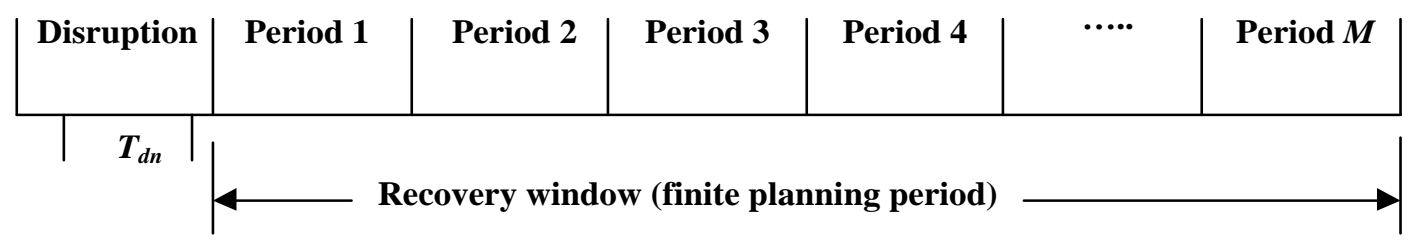

Figure 4: Mechanism of recovery plan for managing sudden disruption

The considered system may face multiple disruptions, one after the other in a series, within its recovery window. In the case of a sudden series of disruptions, the new disruptions may or 
may not affect the recovery plans of the previous disruptions. If a disruption occurs when the ideal plan is running, or after the recovery window of another disruption, then that disruption can be considered as an independent disruption and the recovery plan can be made similar to a single sudden disruption.

If a new sudden disruption occurs during the recovery window of another disruption, a revised recovery plan, incorporating the effect of both disruptions, must be derived, and this makes the algorithm complex. That disruption can be considered as a dependent disruption. It is a continuous process that must be dealt with on a real-time basis. This can be done by updating some of the parameters in the same mathematical model, for example, the newly disrupted plant, the start time of the disruption, the disruption duration, the quantity produced before starting the revised plan and the demand to be filled, to represent the changed scenario in order to re-optimize the plan for the current disruption. After every disruption, the plan is revised for a finite period in the future, for as long as disruptions occur in the system.

Our proposed heuristics for reactive mitigation are capable of dealing with following:

(i) single occurrence of sudden disruption,

(ii) a series of sudden independent disruptions, and

(iii) a series of a mix of independent and dependent disruptions.

In this study, the three different policies for managing a sudden disruption are considered. We assume that both manufacturer and customer agree with these policies. The policies are as follows:

i. Back orders: if a production quantity is lost, the portion of demand that cannot be filled at the scheduled time, but that will be delivered at a later date when available, is known as the back orders quantity (Paul et al., 2014a). This policy uses the spare capacity of manufacturing plants to produce the unfulfilled demand.

ii. Lost sales: if the system is not capable of filling demand after a disruption and customers will not wait for stock to be replenished, demand is lost (Paul et al., 2014a).

iii. Outsourcing: if the production system is not capable of filling the demand on time, as an alternative to lost sales a manufacturer may want to purchase some items from another company at a higher cost and then deliver those items to the customers (Chen and Xiao, 2015). 
In this paper, for reactive mitigation planning, we consider different policies such as back orders, lost sales and/or outsourcing, with an objective of minimizing the total cost. We consider lost sales and outsourcing policy are substitute of each other because these options are applicable when the system is not capable of recovering with its spare capacity and the back orders option. In such case, the management will have two options, either lost sales or outsourcing. Sometimes, outsourcing is not a good option because it may involve additional cost resulting in a higher cost than the lost sales. So outsourcing option will be utilized, instead of lost sales, in the recovery plan only when the outsourcing cost is less than the lost sales cost.

\subsection{Notations used in study}

The following notations are used in this study to formulate the mathematical model.

$i \quad$ Plant index

$j \quad$ DC index

$k \quad$ Retailer index

I Number of plants

$J \quad$ Number of DCs

$K \quad$ Number of retailers

$P_{i} \quad$ Production quantity of plant $i$ under ideal conditions

$C P_{i} \quad$ Maximum production capacity of plant $i$ under ideal conditions

$C D_{j} \quad$ Maximum handling capacity of $\mathrm{DC} j$

$X_{i j} \quad$ Transportation quantity from plant $i$ to DC $j$ under ideal conditions

$Y_{j k} \quad$ Transportation quantity from DC $j$ to retailer $k$ under ideal conditions

$D_{k} \quad$ Demand of retailer $k$

$p_{i} \quad$ Production cost per unit at plant $i$ (\$ per unit)

$H_{1 i} \quad$ Holding cost per unit per period at plant $i$ (\$ per unit per period)

$H_{2 j} \quad$ Handling cost per unit at DC $j$ (\$ per unit)

$H_{3 k} \quad$ Holding cost per unit per period at retailer $k$ (\$ per unit per period)

$T_{1 i j} \quad$ Transportation cost per unit from plant $i$ to DC $j$ (\$ per unit)

$T_{2 j k} \quad$ Transportation cost per unit from DC $j$ to retailer $k$ (\$ per unit)

$O C_{j} \quad$ Operating cost of $\mathrm{DC} j$ (\$ per period)

$S C_{i} \quad$ Spare capacity of plant $i$

$t_{n} \quad$ Start time of disruption at the $n^{\text {th }}$ plant as fraction of period 
$T_{d n} \quad$ Disruption duration for the $n^{\text {th }}$ plant as fraction of period

$P_{i m}^{\prime} \quad$ Production quantity after disruption at plant $i$ in period $m$

$X_{i j m}^{\prime} \quad$ Transportation quantity from plant $i$ to DC $j$ after disruption in period $m$

$Y_{j k m}^{\prime} \quad$ Transportation quantity from DC $j$ to retailer $k$ after disruption in period $m$

$D_{k m}^{\prime} \quad$ Quantity received by retailer $k$ after disruption in period $m$

$L \quad$ Lost sales cost per unit (\$ per unit)

$B \quad$ Back orders cost per unit per period (\$ per unit per period)

$S \quad$ Outsourcing cost per unit (\$ per unit)

$M \quad$ Number of periods in recovery window

\subsection{Assumptions of study}

In this study, we make the following assumptions.

i. A single type of item is produced in the system.

ii. There is no inventory buffer and safety stock in the system.

iii. In predictive mitigation, only changes in demand are predicted.

iv. Disruptions at different manufacturing plants are considered to be independent.

v. The reactive mitigation plan considers back orders, lost sales and/or outsourcing policies to recover from a sudden disruption. Both manufacturer and customers agree with these policies.

vi. The number of periods in a recovery window is decided by the management of the manufacturing organization.

To develop and analyze mitigation plans in a supply chain, we consider that a single item is produced in the system, as has many other researchers in the literature. We also assume that there is no inventory buffer and safety stock as we consider that the cost for these is too expensive. In predictive mitigation planning, we predict the changes in base forecasted demand only by using the FIS tool. In reactive mitigation planning, we consider that sudden disruptions can happen at differing manufacturing plants, but they are independent, as they are located in different geographical area. To make the disruption recovery meaningful in practice, the reactive mitigation plan will be generated after each disruption is experienced by the system. In other words, the reactive mitigation plan is generated on a real-time basis. We consider back orders (Paul et al., 2014a), lost sales (Paul et al., 2014a), and outsourcing (Chen and Xiao, 2015) policies to recover from a sudden disruption, which is also widely used in both literature and practice. Finally, we assume that the number of periods in a 
recovery window is decided by the management of each manufacturing organization, and that both other manufacturers and customers agree with this.

\section{MODEL FORMULATION}

In this section, we formulate the mathematical model for both an ideal and a disrupted supply chain system. The ideal plan is updated if there are any changes in the data and is also revised according to any prediction of future changes for a finite planning period. In the case of managing a disruption, the model is re-formulated to incorporate the effect of a disruption and the production and distribution plan is revised for a finite planning period. After the recovery window, the production and distribution plan reverts to its ideal plan.

\subsection{Formulation of ideal plan}

In this section, different costs are calculated to formulate the mathematical model for the ideal system. The production cost is determined as the per unit production cost multiplied by the production quantity, the average holding cost as the unit holding cost multiplied by the total inventory, the transportation cost as the unit transportation cost multiplied by the transportation quantity, the total operating cost as the sum of the operating cost of each DC, and the handling cost of distribution is the unit handling cost multiplied by the total handling quantity. Finally, the different costs are summed to obtain the objective function to be minimized subject to capacity, distribution and demand constraints, where $P_{i}, X_{i j}$ and $Y_{j k}$ are decision variables. The final mathematical model is considered as a constrained programming problem.

\section{Costs at plant}

Production cost $=\sum_{i=1}^{I} p_{i} P_{i}$

Average holding cost $=\sum_{i=1}^{I} \frac{1}{2} H_{1 i} P_{i}$

Transportation cost $=\sum_{j=1}^{J} \sum_{i=1}^{I} T_{1 i j} X_{i j}$

\section{Costs at DCs}

Operating cost $=\sum_{j=1}^{J} O C_{j}$

Handling cost $=\sum_{j=1}^{J} \sum_{i=1}^{I} H_{2 j} X_{i j}$

Transportation cost $=\sum_{k=1}^{K} \sum_{j=1}^{J} T_{2 j k} Y_{j k}$ 


\section{Costs at retailer}

Average holding cost $=\sum_{k=1}^{K} \frac{1}{2} H_{3 k} D_{k}$

\section{Objective function}

The total supply chain cost (TC), which is the objective function, is derived using equations (1) to (7) and equals to the total plant cost + total DC cost + total retailer cost,

$$
\begin{aligned}
T C=\sum_{i=1}^{I} p_{i} P_{i} & +\sum_{i=1}^{I} \frac{1}{2} H_{1 i} P_{i}+\sum_{J=1}^{J} \sum_{i=1}^{I} T_{1 i j} X_{i j}+\sum_{j=1}^{J} O C_{j}+\sum_{j=1}^{J} \sum_{i=1}^{I} H_{2 j} X_{i j}+\sum_{k=1}^{K} \sum_{j=1}^{J} T_{2 j k} Y_{j k} \\
& +\sum_{k=1}^{K} \frac{1}{2} H_{3 k} D_{k}
\end{aligned}
$$

Here, $P_{i}, X_{i j}$ and $Y_{j k}$ are decision variables, subject to the following constraints.

$$
\begin{aligned}
& P_{i} \leq C P_{i} ; \forall i \\
& P_{i}=\sum_{j=1}^{J} X_{i j} ; \forall i \\
& \sum_{i=1}^{I} X_{i j}=\sum_{k=1}^{K} Y_{j k} ; \forall j \\
& \sum_{i=1}^{I} X_{i j} \leq C D_{j} ; \forall j \\
& \sum_{j=1}^{J} Y_{j k}=D_{k} ; \forall k \\
& \sum_{i=1}^{I} P_{i}=\sum_{k=1}^{K} D_{k} \\
& P_{i}, X_{i j} \text { and } Y_{j k} \geq 0 \text { and integer; } \forall i, j, k
\end{aligned}
$$

The production quantity of each plant is less than or equal to the maximum capacity of that plant (equation (9)), the constraints for distribution from the plant to DCs and DCs to retailers are equations (10) and (11) respectively, the capacity constraints of the DCs equation (12), the demand of the retailers equation (13) and total production is equal to total demand (equation (14)), while constraint in equation (15) is a non-negativity and integer condition of the decision variables.

\subsection{Formulation for update and predictive mitigation plan}

The formulations for the updated and predictive mitigation planning approaches are presented in Appendix A.

\subsection{Formulation for reactive mitigation plan}

In this section, a mathematical model for revising the production and distribution plan for a finite planning period after the occurrence of a production disruption, with the objective of minimizing the total supply chain cost, is developed. As the recovery strategy involves back 
orders, outsourcing and lost sales options, there are additional cost equations for them. The back orders cost is determined as the unit back orders cost multiplied by the number of back orders units and the time delay (Paul et al., 2015b), the lost sales cost is the unit lost sales cost multiplied by the number of lost sales units (Paul et al., 2015b) and the outsourcing cost is the quantity outsourced by the unit purchase cost.

If there is a disruption at the $n^{\text {th }}$ plant for a duration of $T_{d n}$ with a start time of $t_{n}$, the production quantity loss after a single disruption can be determined using equations (16) and (17).

If $t_{n}+T_{d n}<\frac{P_{n}}{C P_{n}}$

$$
D^{\prime}=C P_{n} * T_{d n}-\min \left\{\sum_{i=1}^{I} S C_{i}, \sum_{i=1}^{I} C P_{i} *\left(1-t_{n}-T_{d n}\right)\right\}
$$

$$
\begin{aligned}
& \text { If } t_{n}+T_{d n}>\frac{P_{n}}{C P_{n}} \\
& \qquad D^{\prime}=C P_{n} *\left(\frac{P_{n}}{C P_{n}}-t_{n}\right)-\min \left\{\sum_{i=1}^{I} S C_{i}, \sum_{i=1}^{I} C P_{i} *\left(1-t_{n}-T_{d n}\right)\right\}
\end{aligned}
$$

As this quantity needs to be filled during the recovery window, we consider back orders, lost sales and outsourcing options so that the total supply chain cost during this time can be minimized.

\section{Costs at plant}

Production cost $=\sum_{m=1}^{M} \sum_{i=1}^{I} p_{i} P_{i m}^{\prime}$

Average holding cost $=\sum_{m=1}^{M} \sum_{i=1}^{I} \frac{1}{2} H_{1 i} P_{i m}^{\prime}$

Transportation cost $=\sum_{m=1}^{M} \sum_{j=1}^{J} \sum_{i=1}^{I} T_{1 i j} X_{i j m}^{\prime}$

\section{Costs at DCs}

Operating cost $=M * \sum_{j=1}^{J} O C_{j}$

Handling cost $=\sum_{m=1}^{M} \sum_{j=1}^{J} \sum_{i=1}^{I} H_{2 j} X_{i j m}^{\prime}$

Transportation cost $=\sum_{m=1}^{M} \sum_{k=1}^{K} \sum_{j=1}^{J} T_{2 j k} Y_{j k m}^{\prime}$

\section{Costs at retailer}

Average holding cost $=\sum_{m=1}^{M} \sum_{k=1}^{K} \frac{1}{2} H_{3 k} D_{k m}^{\prime}$ 
Back orders cost $=B * \sum_{m=1}^{M}$ Units delay at period $m *$ delay $_{m}$

$=B *\left[\sum_{m=1}^{M} m\left(\sum_{i=1}^{I} P_{i m}^{\prime}-\sum_{i=1}^{I} P_{i}\right)\right]$

Outsourcing cost

$=S *\left(M \sum_{i=1}^{I} P_{i}+D^{\prime}-\sum_{m=1}^{M} \sum_{i=1}^{I} P_{i m}^{\prime}\right)$

Lost sales cost

$=L *\left(M \sum_{i=1}^{I} P_{i}+D^{\prime}-\sum_{m=1}^{M} \sum_{i=1}^{I} P_{i m}^{\prime}\right)$

If $S \leq L$, then the lost sales cost $=0$, otherwise the outsourcing cost $=0$.

The total supply chain cost $(T C)$, which is the objective function, is derived using equations (18) to (28) and equals the total plant cost + total DC cost + total retailer cost + back orders cost + outsourcing cost + lost sales cost, where $P_{i m}^{\prime}, X_{i j m}^{\prime}, Y_{j k m}^{\prime}$ and $D_{k m}^{\prime}$ are decision variables.

$P_{\text {im }}^{\prime} \leq C P_{i} ; \forall i, m$

$P_{i m}^{\prime}=\sum_{j=1}^{J} X_{i j m}^{\prime} ; \forall i, m$

$\sum_{i=1}^{I} X_{i j m}^{\prime}=\sum_{k=1}^{K} Y_{j k m}^{\prime} ; \forall j, m$

$\sum_{i=1}^{I} X_{i j m}^{\prime} \leq C D_{j, m} ; \forall j, m$

$\sum_{j=1}^{J} Y_{j k m}^{\prime}=D_{k m}^{\prime} ; \forall k, m$

$M \sum_{i=1}^{I} P_{i}+D^{\prime} \geq \sum_{m=1}^{M} \sum_{i=1}^{I} P_{i m}^{\prime}$

$P_{i m}^{\prime}, X_{i j m}^{\prime}, Y_{j k m}^{\prime}$ and $D_{k m}^{\prime} \geq 0$ and integer; $\forall i, j, k, m$

The production quantity of each plant in the revised plan is less than or equal to its maximum capacity in the revised plan (equation (29)), the constraints for distribution from the plant to DCs and from DCs to retailers are equations (30) and (31) respectively, the capacity constraint of the DCs is equation (32), the constraint for the quantity received by each retailer is equation (33), the total lost sales quantity, which should be non-negative, is equation (34) and the non-negativity and integer condition of the decision variables is presented in equation (35).

Proposition 1: for a given $P_{i}, C P_{i}, C D_{j}$ and $D_{k}$, and the $n^{\text {th }}$ disrupted plant, if $B \ll L, S$, the recovery plan will use only the back orders option if $D^{\prime} \leq M \sum_{i=1}^{I} S C_{i}$ and the recovery plan will use both the lost sales/outsourcing and back orders options if $D^{\prime}>M \sum_{i=1}^{I} S C_{i}$.

Proof: let a production disruption occur at the $n^{\text {th }}$ plant starting at period $t_{n}$ with a disruption duration of $T_{d n}$. 
For a disruption, if the spare capacity is greater than or equal to the demand to be filled during the recovery window, as the production system is capable of producing and meeting that demand, the revised plan will utilize only the back orders option as a condition of the existence of only back orders:

$$
\begin{aligned}
& \qquad M \sum_{i=1}^{I} P_{i}+D^{\prime}=\sum_{m=1}^{M} \sum_{i=1}^{I} P_{i m}^{\prime} \\
& \Rightarrow D^{\prime}=\sum_{m=1}^{M} \sum_{i=1}^{I} P_{i m}^{\prime}-M \sum_{i=1}^{I} P_{i} \\
& \Rightarrow D^{\prime} \leq M \sum_{i=1}^{I} C P_{i}-M \sum_{i=1}^{I} P_{i} \text { (using the equation (29)) } \\
& \Rightarrow D^{\prime} \leq M\left(\sum_{i=1}^{I} C P_{i}-\sum_{i=1}^{I} P_{i}\right) \\
& \Rightarrow D^{\prime} \leq M \sum_{i=1}^{I} S C_{i}
\end{aligned}
$$

Therefore, it can be said, that if $D^{\prime} \leq M \sum_{i=1}^{I} S C_{i}$, the system will utilize only the back orders option in the recovery plan.

From the opposite consequence of equation (36), it also can be said that the recovery plan will use both the back orders and lost sales/outsourcing options if $D^{\prime}>M \sum_{i=1}^{I} S C_{i}$.

\section{SOLUTION APPROACHES}

In this section, solution approaches for both ideal and disrupted systems are developed. A standard solution technique for solving the ideal supply chain system is proposed and applied to obtain updated and predictive mitigation plans for changes in the data and future predictions respectively. An efficient heuristic for managing a single disruption in the system is developed and then extended to be implemented for managing multiple disruptions on a real-time basis.

\subsection{Solution approach for generating supply chain plan}

The ideal production and distribution plan is obtained using the branch and bound algorithm of the LINGO optimization software to solve the model for the system, which is a constrained programming problem, and is also applied to obtain updated and predictive mitigation plans.

\subsection{Heuristic for managing single disruption}

A heuristic is designed to obtain the revised plan after an occurrence of a single disruption at any plant. Firstly, both the ideal and disrupted systems are solved using the LINGO optimization software and then the heuristic efficiently solves the disruption management model through the following steps. 
Step 1: Input all the information about production and distribution under ideal conditions.

Step 2: Obtain an ideal production and distribution plan by solving the mathematical model for ideal situations and also determine the spare capacity in each plant.

Step 3: Input a production disruption scenario involving a disrupted plant, disruption start time $\left(t_{n}\right)$ and disruption duration $\left(T_{d n}\right)$.

Step 4: Determine the production plan.

4.1. If $B \leq L, S$ :

4.1.1 If $D^{\prime} \leq M \sum_{i=1}^{I} S C_{i}$, use the spare capacity to revise the plan until the unfilled demand is met;

4.1.2 If $D^{\prime}>M \sum_{i=1}^{I} S C_{i}$, use both the spare capacity and lost sales/outsourcing options;

4.1.2.1 If $L \geq S$, use the outsourcing option and

4.1.2.2 If $L<S$, use the lost sales option.

4.2. If $B>L, S$ :

4.2.1 If $L \geq S$, use the outsourcing option to revise the plan or

4.2.2 If $L<S$, use the lost sales option to revise the plan.

Step 5: Determine the distribution plan.

5.1. If $D^{\prime} \leq M \sum_{i=1}^{I} S C_{i}$, determine the distribution plan by varying only the transportation quantity while using the same path as the ideal plan.

5.2. If $D^{\prime}>M \sum_{i=1}^{I} S C_{i}$, determine the distribution plan by varying only the transportation quantity while using the same path as that obtained from LINGO for $D^{\prime}>M \sum_{i=1}^{I} S C_{i}$.

Step 6: Record the results and determine the different costs.

Step 7: Stop.

\subsection{Proposed heuristic for multiple disruptions}

In this section, the heuristic that was first developed to manage a single disruption is extended to manage multiple production disruptions on a real-time basis. When a disruption occurs, a revised plan can be generated by solving the mathematical model using the proposed heuristic for a single disruption. Then, if another disruption occurs, the plan should be revised again to consider the effects of both disruptions. This can be done by simply updating some of the parameters in the same mathematical model to represent the changed scenario; for example, the newly disrupted plant, start time of the disruption, disruption duration, quantity produced before starting the revised plan and demand to be filled in the revised plan. The 
objective function and constraints are also updated for the changed situation. Therefore, the heuristic for a single disruption can still be used but must be slightly modified for the changed situation to be capable of dealing with a series of disruptions on a real-time basis. In the proposed approach, the heuristic must be run every time a disruption occurs to reoptimize the revised plan whenever there are disruptions in the system.

For a series of disruptions, the production quantity loss after the $s^{\text {th }}$ disruption can be determined using equations (37) and (38).

If $t_{n}+T_{d n}<\frac{P_{n}}{C P_{n}}$

$$
\begin{aligned}
D^{\prime}{ }_{s}=D^{\prime}{ }_{s-1}- & \left(\sum_{m=1}^{l} \sum_{i=1}^{I} P_{i m}^{\prime}-l * \sum_{i=1}^{I} P_{i}\right)+C P_{n} * T_{d n} \\
& -\min \left\{\sum_{i=1}^{I} S C_{i}, \sum_{i=1}^{I} C P_{i} *\left(1-t_{n}-T_{d n}\right)\right\}
\end{aligned}
$$

If $t_{n}+T_{d n}>\frac{P_{n}}{C P_{n}}$

$$
\begin{aligned}
D^{\prime}{ }_{s}=D^{\prime}{ }_{s-1}- & \left(\sum_{m=1}^{l} \sum_{i=1}^{I} P_{i m}^{\prime}-l * \sum_{i=1}^{I} P_{i}\right)+C P_{n} *\left(\frac{P_{n}}{C P_{n}}-t_{n}\right) \\
& -\min \left\{\sum_{i=1}^{I} S C_{i}, \sum_{i=1}^{I} C P_{i} *\left(1-t_{n}-T_{d n}\right)\right\}
\end{aligned}
$$

Here, $l$ is the new disrupted period since the previous disruption.

The main steps in the proposed heuristic for a series of disruptions on a real-time basis can be presented as follows.

Step 1: Input all the information about production and distribution under ideal conditions.

Step 2: Determine the optimal plan under ideal conditions.

Step 3: Input the disruption scenario (disrupted plant, disrupted period since the previous disruption, disruption start time $\left(t_{n}\right)$ and disruption duration $\left.\left(T_{d n}\right)\right)$.

Step 4: Update the loss of production quantity using equations (37) and (38).

Step 5: Revise the production plan for the corresponding disruption using the proposed heuristic developed in Section 4.2.

Step 6: Record and update the optimal production and distribution plan from Step 5 after the disruption occurs. 
Step 7: If there is any other disruption, go to Step 3.

Step 8: Stop.

The heuristic for managing both a single disruption and multiple disruptions is coded in MATLAB R2015b and was executed on an Intel core i7 processor with a $3.40 \mathrm{GHz}$ CPU and a 8.00 GB RAM.

\section{EXPERIMENTATION AND ANALYSIS OF RESULTS}

In this section, we discuss the experiments and results for both the ideal and disrupted systems, and the updated and predictive mitigation plans for a good number of randomly generated test problems. For the disrupted system, the results for both a single disruption and multiple disruptions are analysed. The test problems are solved using both the heuristic, and the branch and bound algorithm of the LINGO optimization software. To judge the quality of the heuristic solutions, we also compare the results obtained from the two different techniques.

\subsection{Experimentation for ideal system}

The following data were considered for an ideal supply chain network.

$$
\begin{gathered}
I=2 ; J=3 ; K=6 ; C P_{i}=[2000,2700] ; D_{k}=[450,500,650,725,800,1000] ; \\
C D_{j}=[2500,2000,1500] ; p_{i}=[19,22] ; H_{1 i}=[1.2,1] ; H_{2 j}=[1.5,1.2,0.8] ; \\
H_{3 k}=[1.5,1.2,0.8,1.75,1,0.9] ; O C_{j}=[10000,15000,8000] \\
T_{1 i j}=\left[\begin{array}{lllll}
3.0 & 4.0 & 5.0 \\
5.0 & 6.0 & 3.0
\end{array}\right] ; T_{2 j k}=\left[\begin{array}{lllll}
6.0 & 2.0 & 4.03 .0 & 6.0 & 4.0 \\
8.0 & 5.0 & 3.02 .0 & 6.0 & 4.0 \\
6.0 & 2.0 & 8.06 .0 & 6.0 & 7.0
\end{array}\right]
\end{gathered}
$$

The ideal system was solved using the branch and bound algorithm of the LINGO optimization software, the generated optimal plan for minimizing the total supply chain cost is presented in Appendix B.

\subsection{Experimentation for updated and predictive mitigation plans}

In this section, we discuss the results for both the updated and predictive mitigation plans using random data.

\subsubsection{Updated plan}

The results for the updated plan are summarized in Appendix C. 


\subsubsection{Predictive mitigation plan}

In this section, we analyse the results for the predictive mitigation approach. We predicted the changes in demand over the base forecast by applying the rule and logic-based FIS that was developed using the fuzzy toolbox of MATLAB R2015b. Then, we revised the plan by solving the mathematical model for the predicted changes in demand data. For this analysis, we predicted the change of demand in the next third period and revised the plan accordingly in a finite planning horizon of three periods by using LINGO. Table 2 presents the range of data that was considered for different predicted events. The pertinent rules developed in FIS tool to relate input and predicted values are presented in Appendix D. Based on the predicted value from the FIS, the equation (39) was used for obtaining the predicted demand value.

predicted demand value $=$ base demand forecast \pm value from FIS

Table 2: Range of data for predictions

\begin{tabular}{|c|c|c|c|c|c|c|}
\hline Event & $\mathrm{NH}$ & NM & NL & PL & $\mathrm{PM}$ & $\mathrm{PH}$ \\
\hline $\begin{array}{l}\text { Demand } \\
\text { fluctuation }\end{array}$ & {$[-1-0.8-0.6]$} & {$\left[\begin{array}{lll}-0.7 & -0.5 & -0.3\end{array}\right]$} & {$[-0.4-0.20]$} & {$\left[\begin{array}{lll}0 & 0.2 & 0.4\end{array}\right]$} & {$\left[\begin{array}{llll}0.3 & 0.5 & 0.7\end{array}\right]$} & {$\left[\begin{array}{llll}0.6 & 0.8 & 1\end{array}\right]$} \\
\hline $\begin{array}{c}\text { Unexpected } \\
\text { incident }\end{array}$ & --- & --- & --- & {$\left[\begin{array}{lll}0 & 0.2 & 0.4\end{array}\right]$} & {$\left[\begin{array}{lll}0.3 & 0.5 & 0.7\end{array}\right]$} & {$\left[\begin{array}{lll}0.6 & 0.8 & 1\end{array}\right]$} \\
\hline $\begin{array}{l}\text { Natural } \\
\text { incident }\end{array}$ & {$[-1-0.8-0.6]$} & {$[-0.7-0.5-0.3]$} & {$[-0.4-0.20]$} & {$\left[\begin{array}{lll}0 & 0.2 & 0.4\end{array}\right]$} & {$\left[\begin{array}{llll}0.3 & 0.5 & 0.7\end{array}\right]$} & {$\left[\begin{array}{lll}0.6 & 0.8 & 1\end{array}\right]$} \\
\hline \multicolumn{7}{|c|}{ NH: negative high, NM: negative medium, NL: negative low } \\
\hline
\end{tabular}

For understanding the process of generating a predictive mitigation plan, one numerical example is presented here. We have predicted the changes in forcasted demand to $[439,593$, $661,734,891,949]$ in the third period of the future by using the developed FIS tool based on information of demand flucation, unexpected incident and natural incident. In the ideal plan, the base forecasted demand was $[450,500,650,725,800,1000]$. So we revised the supply chain plan according to the prediction for the next three periods so that the total cost can be minimized. All of the other parameters were kept constant, as in Section 5.1. We solved the mathematical model that was developed for predictive mitigation plans by using the LINGO optimization software. 


\subsection{Reactive mitigation plan for a sudden single disruption}

For the single disruption problem, we used the same basic data as for the ideal system. For illustrative purposes, sixteen different disruption scenarios were generated and their parameters are presented in Table 3. We classified the disruption start times as early, middle and late, and disruption durations as low, medium and high. As when the disruption start time was in the late range, it was not possible to have a high disruption duration, because the latter was dependent on the former, two scenarios (1-L-H and 2-L-H) were absent from the design of this experiment. Then, we generated 10 random test problems for each scenario by varying the cost data while using the following additional data.

$$
B=10, L=50, S=60 \text {, and } M=2
$$

Table 3: Design of experiment with total of sixteen scenarios

\begin{tabular}{|c|c|c|c|}
\hline Disrupted plant & $\begin{array}{l}\text { Disruption start } \\
\text { time }\end{array}$ & Disruption duration & Scenario name \\
\hline \multirow{3}{*}{1} & \multirow{3}{*}{0.60 (late) } & - & - \\
\hline & & 0.35 (medium) & 1-L-M \\
\hline & & 0.20 (low) & 1-L-L \\
\hline \multirow{3}{*}{1} & \multirow{3}{*}{0.30 (middle) } & 0.55 (high) & 1-M-H \\
\hline & & 0.33 (medium) & 1-M-M \\
\hline & & 0.20 (low) & 1-M-L \\
\hline \multirow{3}{*}{1} & \multirow{3}{*}{0.10 (early) } & 0.70 (high) & 1-E-H \\
\hline & & 0.50 (medium) & 1-E-M \\
\hline & & 0.18 (low) & 1-E-L \\
\hline \multirow{3}{*}{2} & \multirow{3}{*}{0.55 (late) } & - & - \\
\hline & & 0.30 (medium) & 2-L-M \\
\hline & & 0.15 (low) & 2-L-L \\
\hline \multirow{3}{*}{2} & \multirow{3}{*}{0.35 (middle) } & 0.60 (high) & 2-M-H \\
\hline & & 0.40 (medium) & 2-M-M \\
\hline & & 0.10 (low) & 2-M-L \\
\hline \multirow{3}{*}{2} & \multirow{3}{*}{0.05 (early) } & 0.75 (high) & 2-E-H \\
\hline & & 0.30 (medium) & 2-E-M \\
\hline & & 0.12 (low) & 2-E-L \\
\hline
\end{tabular}


The sixteen scenarios in Table 3, each with ten random test problems, were solved using both the heuristic and the LINGO optimization software. We compared the results for all 160 random disruption test problems and the average percentage of deviation between those obtained from the two approaches, calculated by equation (40), was only $0.0007 \%$, which, again, was negligible. Moreover, this may merely have been due to the rounding of the values of the decision variables. It can be said that the results obtained from the two approaches were very consistent.

Average percentage of deviation

$=\frac{1}{N} \sum \frac{\mid \text { Total profit from heuristic-Total profit from LINGO } \mid}{\text { Total profit from LINGO }} \times 100 \%$

Here, $N=$ total number of test problems.

\subsection{Reactive mitigation plans for a series of sudden disruptions}

To demonstrate the usefulness of our proposed heuristic for solving different scenarios with multiple production disruptions, we used the same basic data as for the ideal and disrupted systems presented in Sections 5.1 and 5.3 respectively. In a supply chain system, a series of production disruptions can occur at any plant, one after another, on a real-time basis. The first disruption can be managed using the single disruption approach discussed in the previous section. If another disruption occurs at any plant during the recovery window of the previous one, as this may affect the revised plan of previous disruptions, this plan must be considered as a set of additional restrictions. For experimental purposes, several random disruptions were generated to occur one after another. Table 4 presents cases with different random combinations of a disrupted plant, disruption start times and disruption durations. Although disruptions can happen continuously within a production cycle, we present only five disruptions as a sample representation. The production and distribution plans were revised immediately after each disruption occurred in the system. The problem was solved using the proposed heuristic, and the results after each disruptionfor the total supply chain and the total lost sales cost in the revised plan are presented in Table 5. It was observed that the system utilized both the spare capacity and lost sales options for the first three disruptions for revising the plan, and it was capable of revising the plan by using only the back orders option for the 4 th disruption. After the 5th disruption, the system was capable to recover by utilizing the spare capacity of the other plant so no back orders or lost sales are present in the recovery plan then. 
Table 4: A case problem for a series of disruptions

\begin{tabular}{|c|c|c|c|c|}
\hline $\begin{array}{c}\text { Disruption } \\
\text { number }\end{array}$ & Disrupted plant & $\begin{array}{c}\text { Disrupted } \\
\text { period since } \\
\text { previous } \\
\text { disruption }\end{array}$ & $\begin{array}{c}\text { Disruption start } \\
\text { time }\end{array}$ & $\begin{array}{c}\text { Disruption } \\
\text { duration }\end{array}$ \\
\hline 1 & 1 & -- & 0.05 & 0.85 \\
\hline 2 & 2 & 3 & 0.22 & 0.70 \\
\hline 3 & 2 & 1 & 0.55 & 0.25 \\
\hline 4 & 1 & 4 & 0.35 & 0.30 \\
\hline 5 & 2 & 2 & 0.70 & $\ldots$ \\
\hline$\ldots$ & $\ldots$ & $\ldots$ & $\ldots$ & 0.10 \\
\hline
\end{tabular}

Table 5: Summary of results for a series of disruptions

\begin{tabular}{|c|c|c|c|}
\hline $\begin{array}{c}\text { Disruption } \\
\text { number }\end{array}$ & Total cost $(\$)$ & $\begin{array}{c}\text { Total back orders } \\
\text { cost }(\$)\end{array}$ & $\begin{array}{c}\text { Total lost sales cost } \\
(\$)\end{array}$ \\
\hline 1 & $3,79,990$ & 17,250 & 4,000 \\
\hline 2 & $3,76,240$ & 17,250 & 250 \\
\hline 3 & $3,78,990$ & 17,250 & 0 \\
\hline 4 & $3,17,912$ & 250 & 0 \\
\hline 5 & $3,16,830$ & 0 & $\ldots$ \\
\hline
\end{tabular}

\subsection{Effect of disruption duration in reactive mitigation}

As the disruption duration has a significant impact on the total supply chain, back orders and lost sales costs, its relationships with these factors reanalysed. This section presents a number of studies, in each of which only one variable is changed while the other parameters have the default values of the ideal system presented in Section 5.1. We consider an independent and a single disruption to analyse the effect of disruption duration.

Figures 5, 6 and 7 respectively show the changes in the total supply chain, back orders and lost sales costs for varying disruption durations. In this analysis, the disruption start time is 
kept constant at 0 , and it is observed that the total supply chain cost increases with increasing durations of disruptions at both plants 1 and 2 .

In Figure 5, it is observed that the revised plan uses no additional cost when the disruption durations are less than or equal to 0.20 and 0.30 for disruptions at plant 1 and 2 respectively. This is because the system utilizes the spare capacity of the disrupted period to fill the quantity lost. However, there then is an increasing trend in the total supply chain cost because of the introduction of back orders and lost sales costs in the solutions.

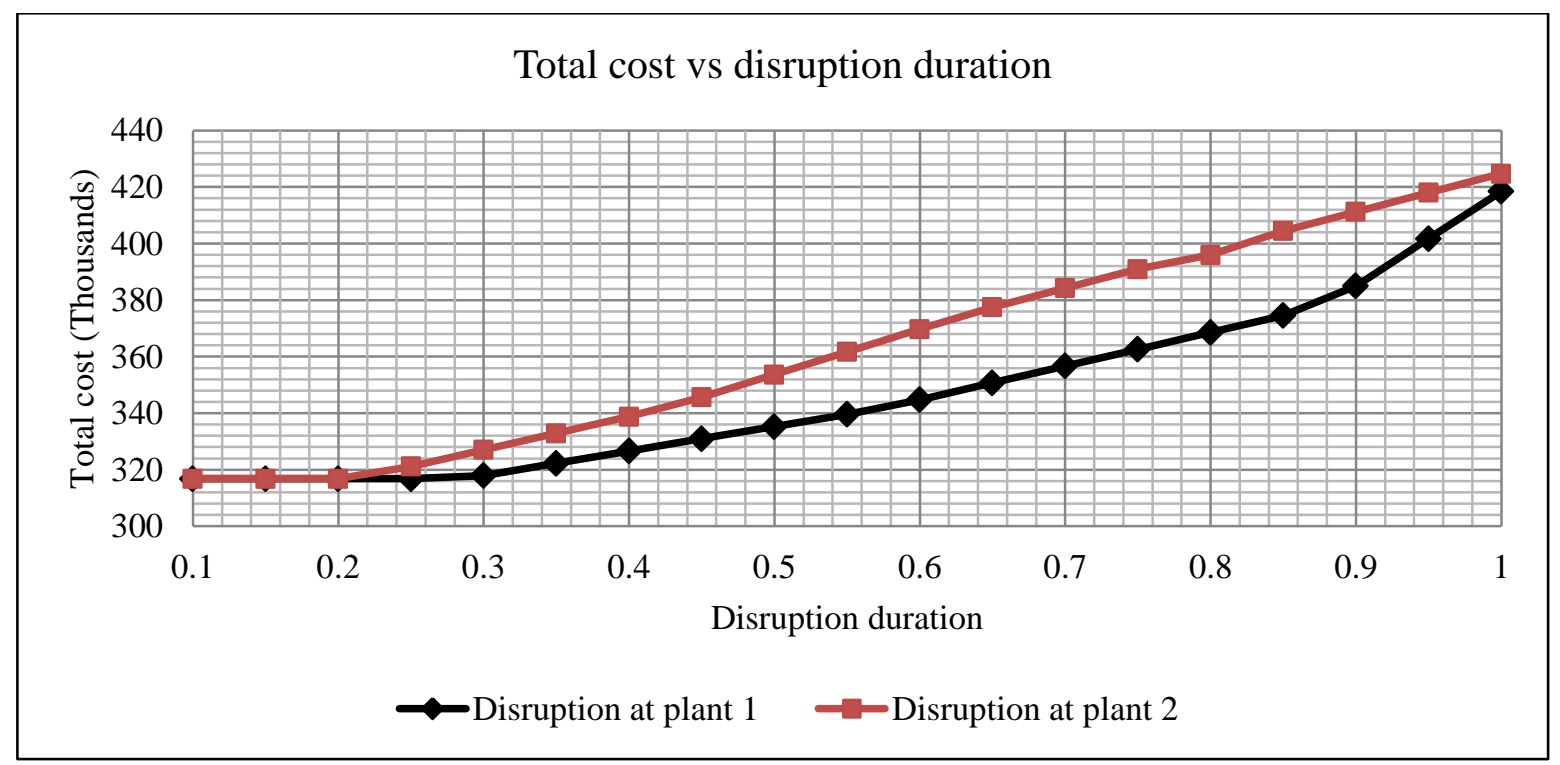

Figure 5: Changes in total supply chain cost for varying disruption durations

Figure 6 presents changes in the back orders cost with different disruption durations. It is observed that they occur in the system after disruption durations of 0.2 and 0.3 at plants 1 and 2 respectively and increase up to those of 0.65 and 0.85 respectively. Then, the lost sales cost appears in the system and the back orders cost becomes a fixed amount.

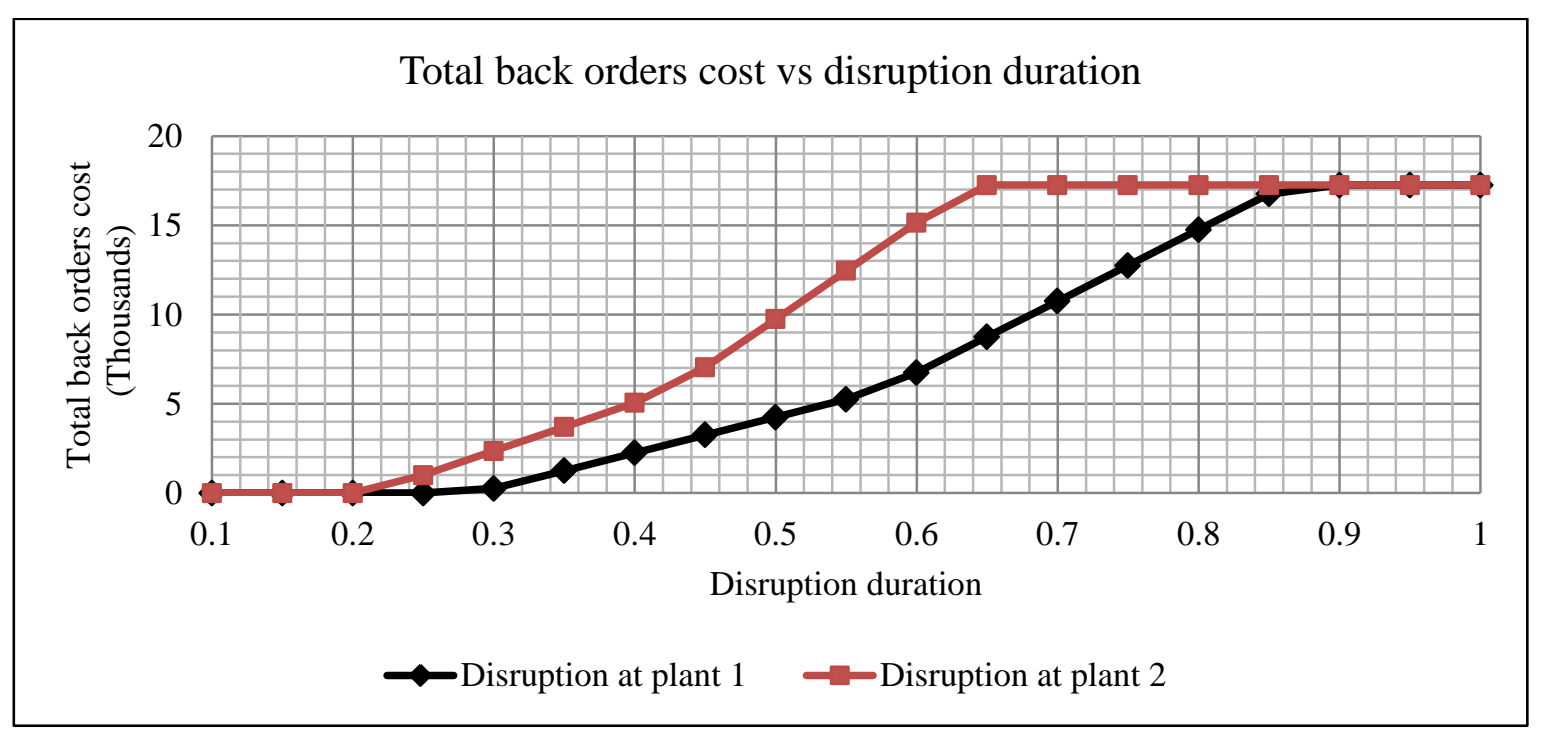

Figure 6: Changes in total back orders cost for varying disruption durations 
Figure 7 presents changes in the lost sales cost for different disruption durations. It is observed that they occur in the system after disruption durations of 0.6 and 0.85 at plants 1 and 2 respectively and then increase. Previously, the system was capable of recovering using only the back orders option.

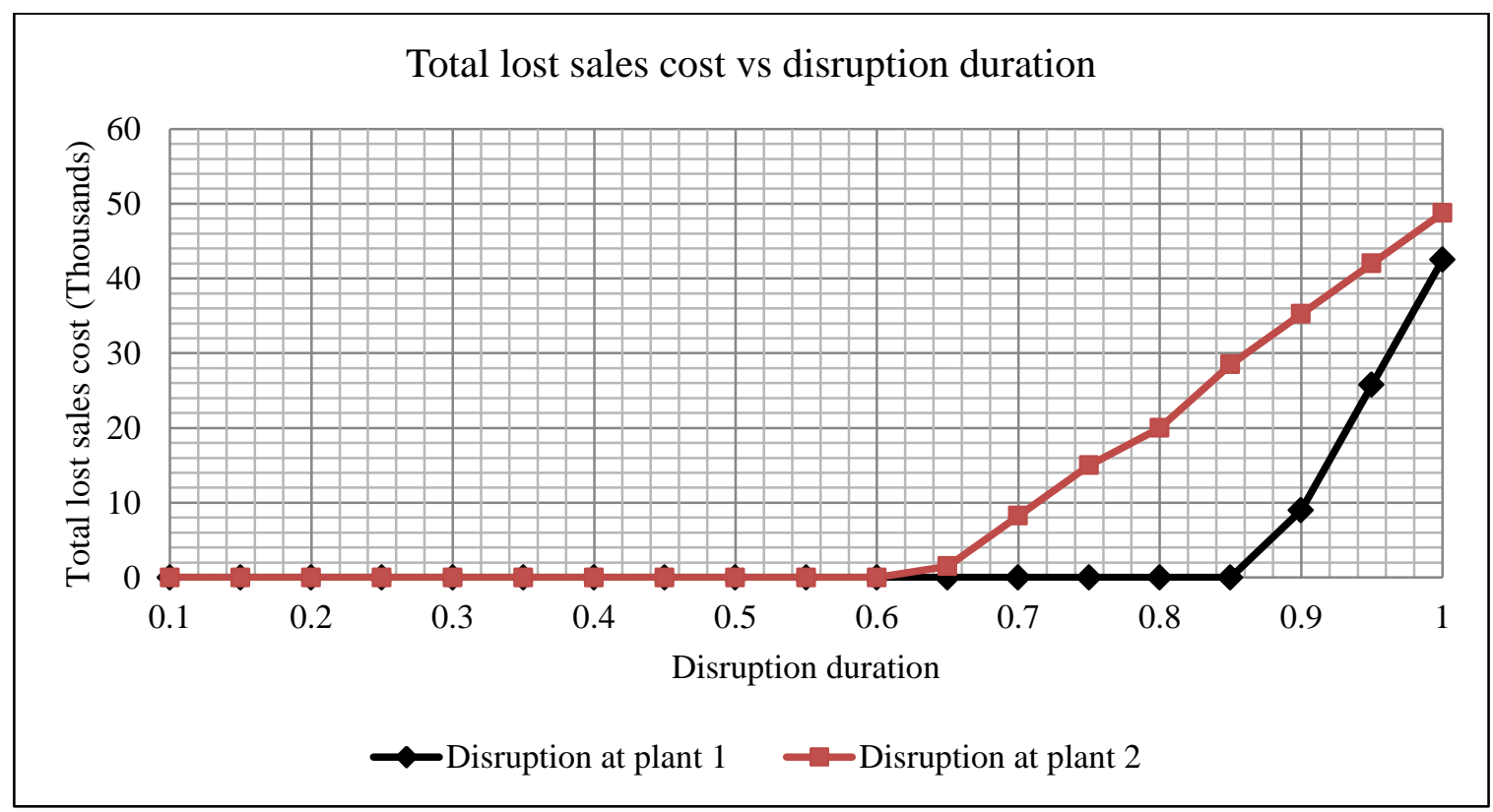

Figure 7: Changes in total lost sales cost for varying disruption durations

\subsection{Effect of $B, L$ and $S$ in reactive mitigation}

The parameters for back orders, lost sales and outsourcing costs are very important for decision making in the reactive mitigation plan. These parameters have significant impacts on different costs. In this section, a sensitivity analysis is performed to analyse the impact of those parameters and how they effect the decisions for the reactive plan. For this analysis, we considered a single and independent sudden disruption at plant 2 with disruption duration 0.8 starting at the beginning of the period. For each of this analysis study, one variable is changed while the other parameters have the default values of the ideal system presented in Section 5.1 .

Figure 8 presents the changes of different costs with back orders cost. We observed, that both total cost and total back orders cost increase with increasing back orders cost. This is because of the increment of per unit back orders cost, and the reactive plan utilizes both back orders and lost sales policies in the recovery plan. We also observed that there is no outsourcing cost because the optimal plan did not use the outsourcing policy, as the outsourcing cost is greater than the lost sales cost. So it was beneficial to use lost sales option. 


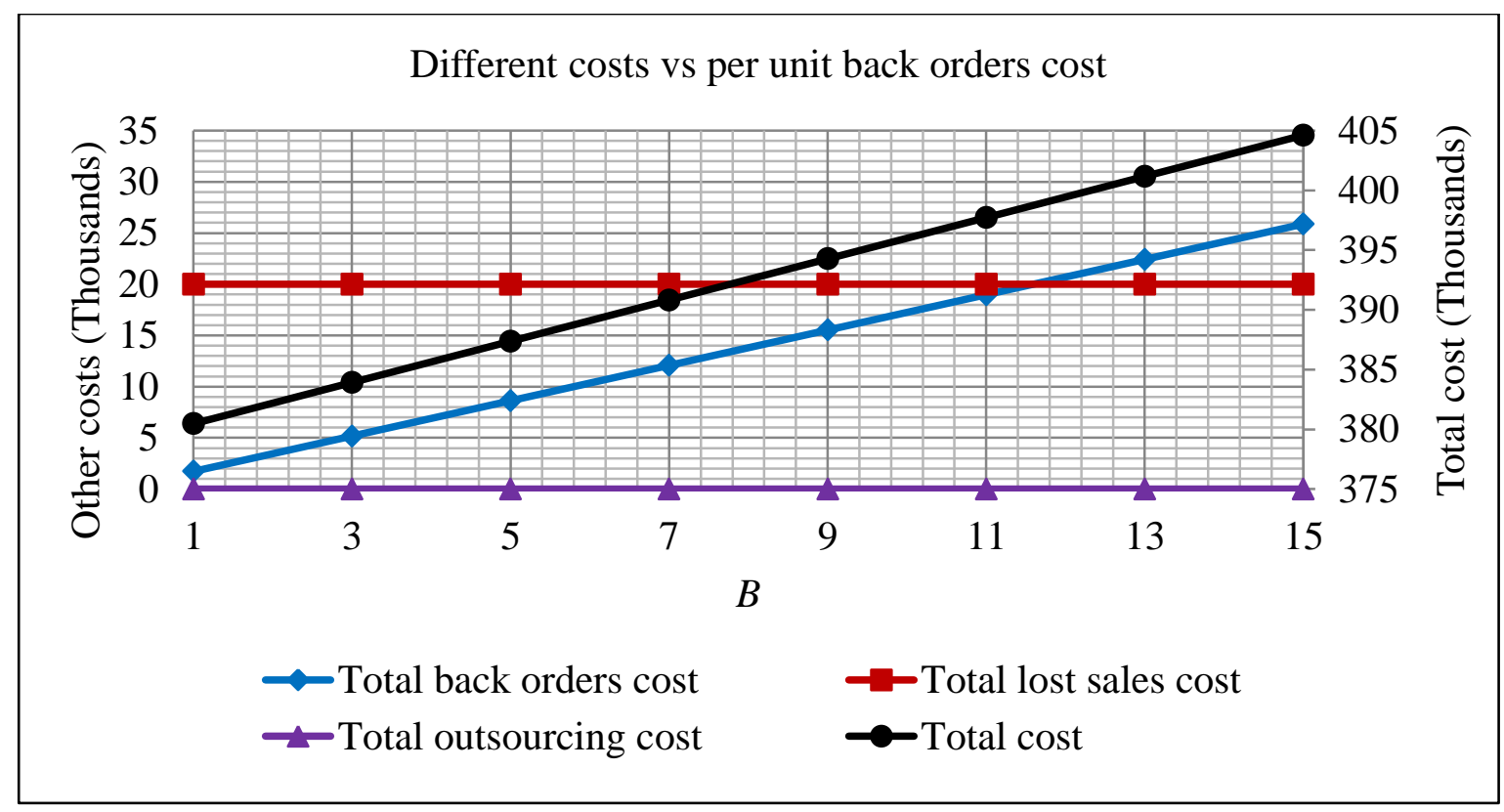

Figure 8: Changes of different costs with $B$ (default: $L=50, S=60$ )

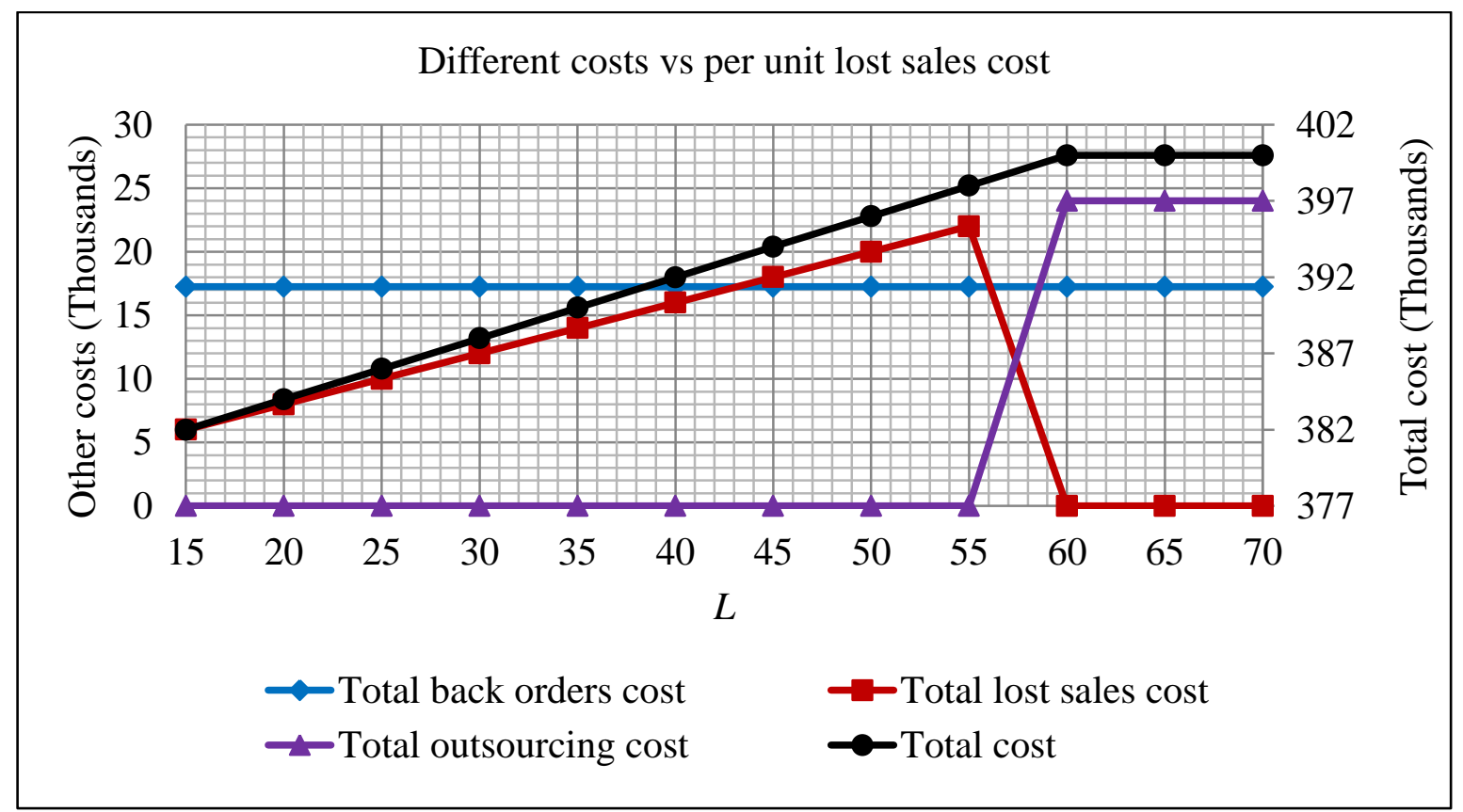

Figure 9: Changes of different costs with $L$ (default: $B=10, S=60$ )

Similarly, Figure 9 presents the effect of per unit lost sales and outsourcing cost on the different costs and a trade-off between lost sales and outsourcing. In this case, the total back orders cost remain unchanged, because the per unit back orders cost remains constant. But, there is a trade-off between total lost sales and outsourcing costs. In Figure 9, we observed that the total lost sales cost increases linearly when per unit lost sales cost is less than the per unit outsourcing cost. When unit lost sales cost is equal or greater than the unit outsourcing cost, the recovery plan utilizes the outsourcing policy, instead of lost sales, and the total lost 
sales cost becomes zero. So management should utilize the outsourcing option, instead of lost sales, only when per unit outsourcing cost less than or equal to per unit lost sales cost.

\section{APPLICABILITY AND VALUE IN PRACTICE}

This paper developed three different approaches for supply chain mitigation:

i. Updated plan for any change in data - the plan was revised if there were any changes in cost and demand data.

ii. Predictive mitigation plan - the changes in demand over the base forecast were predicted in advance by using a rule and logic based FIS prediction tool that is based on information of demand fluctuation, unexpected incidents and natural incidents. The supply chain plan was revised in advance according to that prediction.

iii. Reactive mitigation plan - the supply chain plan was revised after the occurrence of any sudden disruption, which cannot be predicted, on a real-time basis. An efficient heuristic was developed which was capable of dealing with both a single and a series of sudden disruptions on a real-time basis and the supply chain plan was revised just after the occurrence of each disruption, as long as disruptions took place in the system.

In a real-life supply chain environment, a decision maker can easily use any of the three developed approaches to make an efficient and prompt decision quantitatively. Most importantly, this paper developed quantitative approaches for generating both predictive and reactive mitigation plans. In practice, it is common that the forecasted demand can change, but there are limited appropriate tools in the literature to predict that change. The developed FIS tool will help practitioners to predict changes in demand and to so develop better supply chain plans well in advance. This paper developed a rule and logic based FIS tool to predict changes in demand in advance based on information of demand fluctuation, unexpected incidents and natural incidents. The incidents may also affect the plant capacities and transportation routes depending on the nature of incidents. However, the demand is influenced by many external factors, such as consumer behaviour and external incidents, that, that requires sophisticated prediction tools to predict the changes. The nature of changes in plant capacity and transportation is different from the demand. The plant capacities and transportation are influenced mainly by the internal factors such as preventive maintenance and breakdown. To incorporate the effect of such preventive maintenance, the plan can be 
revised using the changed data as discussed in the contribution (i). For breakdown or any other incidents that may disrupt the production suddenly, which is impossible to predict in advance, contributions (iii) to (v) are capable to deal with them. To manage this sudden disruption, this paper developed a reactive mitigation quantitative approach. After the occurrence of a disruption, a decision maker can make an efficient decision by revising the future plan with the help of the developed heuristics for reactive mitigation, whenever a single or series of sudden disruptions occur in the system. We believe that the attractive features of the developed quantitative approaches have added value in the real-life practice of supply chain systems.

\section{CONCLUSIONS}

The main objective of the paper was to develop quantitative approaches for managing any changes in data and for generating both a predictive and reactive mitigation plan. In the case of any changes in data that were known in advance, the supply chain plan was updated according to the changes. For a predictive mitigation plan, a FIS based prediction tool was developed to predict changes in future demand, which are hence not known but predicted and the supply chain plan was revised to obtain a better plan based on the prediction. Random experimentation was conducted by using LINGO for both update and predictive mitigation plans. Finally, this paper developed a quantitative reactive mitigation plan for managing sudden production disruptions that were not known and cannot be predicted. The supply chain plan was revised for a finite period into the future, just after the occurrence of a disruption. The objective is to minimize the effect of the disruption and to return the system to its ideal plan as quickly as possible. For this purpose, we developed an efficient heuristic to obtain the reactive plan. We then developed sixteen different scenarios, each with ten randomly generated disruption test problems, and compared the performances of the heuristic and LINGO for them. It was shown that the average percentage of deviation in the results was only a negligible $0.0007 \%$. We also developed an extended heuristic for managing a series of disruptions, because they are common in real-life supply chain practice. We

presented several numerical examples and experimentations to analyse the results and usefulness of the models.

As the developed approaches have great practical implications, so it can be said that the proposed quantitative and heuristic approaches offer a potentially very useful quantitative means of helping decision makers arrive at prompt and accurate decisions regarding both predictive and reactive mitigation plans. An organization can immediately update/revise its 
plan (i) for any changes in data, (iii) for any future prediction of demand changes, and/or (iii) after the occurrences of any single and/or series of sudden production disruptions in the system.

In future, the proposed approaches can be extended by considering sudden disruptions in supply, distribution and delivery. Additionally, it would be worthwhile to incorporate environmental aspects, such as lowering supply chain costs by reducing travel distances, carbon emission, production costs, product waste, and unplanned activities. Another interesting extension would be to relax the assumption of a single type of item, so as to consider multiple types of items, as well as to analyse the impacts of disruptions on different types of items in a multi-tier supply chain system.

In addition, several aspects could be introduced into the developed approaches, and some of them are listed in the following.

i. Considering safety-stock level and analysing the effect of disruption on safety stock, and determining the optimum level to minimize the effect of a disruption.

ii. Considering lead time factors and analysing the effect of disruption on lead time and disruption recovery.

iii. Considering different shipment policies, such as the multiple-lot for lot, including equal-sized shipment policy, geometric shipment policy and mixtures of them.

\section{ACKNOWLEDGMENT}

The authors express their sincere gratitude and thanks to the reviewers for their positive, constructive and helpful comments and suggestions that helped to improve the manuscript.

\section{REFERENCES}

1. Ahmed, I., Sultana, I., Paul, S. K., \& Azeem, A. (2013). Employee performance evaluation: a fuzzy approach. International Journal of Productivity and Performance Management, 62(7), 718-734.

2. Cavallo, A., Cavallo, E., \& Rigobon, R. (2014). Prices and supply disruptions during natural disasters. Review of Income and Wealth, 60(S2), S449-S471.

3. Chen, K., \& Xiao, T. (2015). Outsourcing strategy and production disruption of supply chain with demand and capacity allocation uncertainties. International Journal of Production Economics, 170, 243-257.

4. Chopra, S., Reinhardt, G., and Mohan, U. (2007). The importance of decoupling recurrent and disruption risks in a supply chain. Naval Research Logistics, 54(5), 544-555. 
5. Craighead, C. W., Blackhurst, J., Rungtusanatham, M. J., \& Handfield, R. B. (2007). The severity of supply chain disruptions: design characteristics and mitigation capabilities. Decision Sciences, 38(1), 131-156.

6. Eisenstein, D. D. (2005). Recovering Cyclic Schedules Using Dynamic Produce-Up-To Policies. Operations Research, 53(4), 675-688.

7. Fahimnia, B., Tang, C. S., Davarzani, H., \& Sarkis, J. (2015). Quantitative models for managing supply chain risks: A review. European Journal of Operational Research, 247(1), 1-15.

8. Gallego, G. (1994). When is a base stock policy optimal in recovering disrupted cyclic schedules? Naval Research Logistics, 41(3), 317-333.

9. Giunipero, L. C., and Eltantawy, R. A. (2004). Securing the upstream supply chain: a risk management approach. International Journal of Physical Distribution \& Logistics Management, 34(9), 698-713.

10. Hasan, M. M., Shohag, M. A. S., Azeem, A., \& Paul, S. K. (2015). Multiple criteria supplier selection: a fuzzy approach. International Journal of Logistics Systems and Management, 20(4), 429-446.

11. Heydari, J. (2014). Supply chain coordination using time-based temporary price discounts. Computers \& Industrial Engineering, 75, 96-101.

12. Hishamuddin, H., Sarker, R. A., and Essam, D. (2012). A disruption recovery model for a single stage production-inventory system. European Journal of Operational Research, 222(3), 464-473.

13. Hishamuddin, H., Sarker, R. A., and Essam, D. (2013). A recovery model for a twoechelon serial supply chain with consideration of transportation disruption. Computers \& Industrial Engineering, 64(2), 552-561.

14. Hishamuddin, H., Sarker, R. A., and Essam, D. (2014). A recovery mechanism for a two echelon supply chain system under supply disruption. Economic Modelling, 38, 555-563.

15. Hult, G. T. M., Craighead, C. W., \& Ketchen Jr, D. J. (2010). Risk uncertainty and supply chain decisions: a real options perspective. Decision Sciences, 41(3), 435-458.

16. Li, Z., Xu, S. H., and Hayya, J. (2004). A periodic-review inventory system with supply interruptions. Probability in the Engineering \& Informational Sciences, 18(1), 33-53.

17. Mohebbi, E., and Hao, D. (2008). An inventory model with non-resuming randomly interruptible lead time. International Journal of Production Economics, 114(2), 755-768.

18. Paul, S. K., Azeem, A., Sarker, R., \& Essam, D. (2014). Development of a production inventory model with uncertainty and reliability considerations. Optimization and Engineering, 15(3), 697-720.

19. Paul, S. K., \& Azeem, A. (2010). Selection of the optimal number of shifts in fuzzy environment: manufacturing company's facility application. Journal of Industrial Engineering and Management, 3(1), 54-67.

20. Pal, B., Sana, S. S., and Chaudhuri, K. (2012). A three layer multi-item productioninventory model for multiple suppliers and retailers. Economic Modelling, 29(6), 27042710.

21. Paul, S. K., Sarker, R., and Essam, D. (2013). A Disruption Recovery Model in a Production-Inventory System with Demand Uncertainty and Process Reliability. Lecture Notes in Computer Science, 8104, 511-522. Springer Berlin Heidelberg. 
22. Paul, S. K., Sarker, R., and Essam, D. (2014a). Real time disruption management for a two-stage batch production-inventory system with reliability considerations. European Journal of Operational Research, 237(1), 113-128.

23. Paul, S. K., Sarker, R., and Essam, D. (2014b). Managing real-time demand fluctuation under a supplier-retailer coordinated system. International Journal of Production Economics, 158, 231-243.

24. Paul, S. K., Sarker, R. A., \& Essam, D. L. (2014c). Managing supply disruption in a three-tier supply chain with multiple suppliers and retailers. In 2014 IEEE International Conference on Industrial Engineering and Engineering Management (IEEM), pp. 194198, 9-12 December, Selangor, Malaysia.

25. Paul, S. K. (2015). Supplier selection for managing supply risks in supply chain: a fuzzy approach. The International Journal of Advanced Manufacturing Technology, 79(1-4), 657-664.

26. Paul, S. K., Sarker, R., \& Essam, D. (2015a). Managing disruption in an imperfect production-inventory system. Computers \& Industrial Engineering, 84, 101-112.

27. Paul, S. K., Sarker, R., \& Essam, D. (2015b). A disruption recovery plan in a three-stage production-inventory system. Computers \& Operations Research, 57, 60-72.

28. Paul, S. K., Sarker, R., \& Essam, D. (2016a). Managing risk and disruption in productioninventory and supply chain systems: A review. Journal of Industrial \& Management Optimization, 12(3), 1009-1029.

29. Paul, S. K., Sarker, R., \& Essam, D. (2016b). A reactive mitigation approach for managing supply disruption in a three-tier supply chain. Journal of Intelligent Manufacturing, in press, 1-17. doi: 10.1007/s10845-016-1200-7

30. Petrovic, D., Xie, Y., Burnham, K., and Petrovic, R. (2008). Coordinated control of distribution supply chains in the presence of fuzzy customer demand. European Journal of Operational Research, 185(1), 146-158.

31. Purnomo, H. D., Wee, H. M., \& Praharsi, Y. (2012). Two inventory review policies on supply chain configuration problem. Computers \& Industrial Engineering, 63(2), 448455.

32. Qi, L., Shen, Z. J. M., and Snyder, L. V. (2009). A continuous- review inventory model with disruptions at both supplier and retailer. Production \& Operations Management, 18(5), 516-532.

33. Qi, X., Bard, J. F., \& Yu, G. (2004). Supply chain coordination with demand disruptions. Omega, 32(4), 301-312.

34. Reza Nasiri, G., Zolfaghari, R., \& Davoudpour, H. (2014). An integrated supply chain production-distribution planning with stochastic demands. Computers \& Industrial Engineering, 77, 35-45.

35. Ross, A. M., Rong, Y., and Snyder, L. V. (2008). Supply disruptions with time-dependent parameters. Computers \& Operations Research, 35(11), 3504-3529.

36. Sana, S. S. (2011). A production-inventory model of imperfect quality products in a three-layer supply chain. Decision Support Systems, 50(2), 539-547.

37. Sana, S. S. (2012). A collaborating inventory model in a supply chain. Economic Modelling, 29(5), 2016-2023. 
38. Snyder, L. V., Atan, Z., Peng, P., Rong, Y., Schmitt, A. J., \& Sinsoysal, B. (2016). OR/MS models for supply chain disruptions: A review. IIE Transactions, 48(2) pp. 89109.

39. Syntetos, A. A., Babai, Z., Boylan, J. E., Kolassa, S., \& Nikolopoulos, K. (2016). Supply chain forecasting: Theory, practice, their gap and the future. European Journal of Operational Research, 252(1), 1-26.

40. Sodhi, M. S., and Chopra, S. (2004). Managing risk to avoid supply-chain breakdown. MIT Sloan management review, 46(1), 53-61.

41. Supply Chain Resilience Report, (2015), BCI survey of resilience professionals, Archived from "http://www.bcifiles.com/bci-supply-chain-resilience-2015.pdf" on 01/03/2016.

42. Tang, L. C., \& Lee, L. H. (2005). A simple recovery strategy for economic lot scheduling problem: A two-product case. International Journal of Production Economics, 98(1), 97107.

43. Tomlin, B. (2006). On the value of mitigation and contingency strategies for managing supply chain disruption risks. Management Science, 52(5), 639-657.

44. Unnikrishnan, A., \& Figliozzi, M. (2011). Online Freight Network Assignment Model with Transportation Disruptions and Recourse. Transportation Research Record: Journal of the Transportation Research Board, 2224(1), 17-25.

45. Wilson, M. C. (2007). The impact of transportation disruptions on supply chain performance. Transportation Research Part E: Logistics \&Transportation Review, 43(4), 295-320.

46. Wu, T., Blackhurst, J., and O'grady, P. (2007). Methodology for supply chain disruption analysis. International Journal of Production Research, 45(7), 1665-1682.

47. Xu, R., and Zhai, X. (2010). Manufacturer's coordination mechanism for single-period supply chain problems with fuzzy demand. Mathematical \& Computer Modelling, 51(5), 693-699.

48. Xia, Y., Yang, M. H., Golany, B., Gilbert, S. M., and Yu, G. (2004). Real-time disruption management in a two-stage production and inventory system. IIE Transactions, 36(2), 111-125.

49. Yang, J., Qi, X., \& Yu, G. (2005). Disruption management in production planning. Naval Research Logistics, 52(5), 420-442.

50. Zhou, C., Zhao, R., and Tang, W. (2008). Two-echelon supply chain games in a fuzzy environment. Computers \& Industrial Engineering, 55(2), 390-405. 


\section{Appendix A}

\section{Formulation of updated and predictive mitigation plans}

$F \quad$ Number of periods in finite planning horizon

$P_{i f} \quad$ Production quantity of plant $i$ at period $f$

$C P_{i f} \quad$ Maximum production capacity of plant $i$ at period $f$

$C D_{j f} \quad$ Maximum handling capacity of DC $j$ at period $f$

$X_{i j f} \quad$ Transportation quantity from plant $i$ to $\mathrm{DC} j$ at period $f$

$Y_{j k f} \quad$ Transportation quantity from DC $j$ to retailer $k$ at period $f$

$D_{k f} \quad$ Demand of retailer $k$ at period $f$

$p_{i f} \quad$ Production cost per unit at plant $i$ at period $f$

$H_{1 \text { if }} \quad$ Holding cost per unit per period at plant $i$ at period $f$

$H_{2 j f} \quad$ Handling cost per unit at DC $j$ at period $f$

$H_{3 k f} \quad$ Holding cost per unit per period at retailer $k$ at period $f$

$T_{1 i j f} \quad$ Transportation cost per unit from plant $i$ to DC $j$ at period $f$

$T_{2 j k f}$ Transportation cost per unit from DC $j$ to retailer $k$ at period $f$

$O C_{j f} \quad$ Operating cost of DC $j$ at period $f$

\section{Costs at plant}

Production cost $=\sum_{f=1}^{F} \sum_{i=1}^{I} p_{i f} P_{i f}$

Average holding cost $=\sum_{f=1}^{F} \sum_{i=1}^{I} \frac{1}{2} H_{1 i f} P_{i f}$

Transportation cost $=\sum_{f=1}^{F} \sum_{j=1}^{J} \sum_{i=1}^{I} T_{1 i j f} X_{i j f}$

\section{Costs at DCs}

Operating cost $=\sum_{f=1}^{F} \sum_{j=1}^{J} O C_{j f}$

Handling cost $=\sum_{f=1}^{F} \sum_{j=1}^{J} \sum_{i=1}^{I} H_{2 j f} X_{i j f}$

Transportation cost $=\sum_{f=1}^{F} \sum_{k=1}^{K} \sum_{j=1}^{J} T_{2 j k f} Y_{j k f}$

\section{Cost at retailer}

Average holding cost $=\sum_{f=1}^{F} \sum_{k=1}^{K} \frac{1}{2} H_{3 k f} D_{k f}$

\section{Objective function}

The total supply chain cost $(T C)$, which is the objective function, is derived using equations (A1) - (A7), and equals the total plant cost + total DC cost + total retailer cost, with $P_{i f}, X_{i j f}$ and $Y_{j k f}$ decision variables, subject to the following constraints. 
$P_{i f} \leq C P_{i f} ; i, f$

$P_{i f}=\sum_{j=1}^{J} X_{i j f} ; \forall i, f$

$\sum_{i=1}^{I} X_{i j f}=\sum_{k=1}^{K} Y_{j k f} ; \forall j, f$

$\sum_{i=1}^{I} X_{i j f} \leq C D_{j f} ; \forall j, f$

$\sum_{f=1}^{F} \sum_{i=1}^{I} P_{i f}=\sum_{f=1}^{F} \sum_{k=1}^{K} D_{k f}$

$P_{i f}, X_{i j f}$ and $Y_{j k f} \geq 0$ and integer; $\forall i, j, k, f$

\section{Appendix B}

Table B1: Optimal production and distribution plan for the ideal system

\begin{tabular}{|c|c|c|c|c|c|c|c|c|c|c|c|c|}
\hline \multirow{2}{*}{$\begin{array}{c}\text { Production } \\
\text { plan }\end{array}$} & \multicolumn{10}{|c|}{ Distribution plan } \\
\cline { 3 - 14 } & & \multicolumn{3}{|c|}{ DC } & & \multicolumn{7}{|c|}{ Retailer } \\
\hline $\mathrm{P}_{1}$ & $\mathrm{P}_{2}$ & Plant & 1 & 2 & 3 & $\mathrm{DC}$ & 1 & 2 & 3 & 4 & 5 & 6 \\
\hline \multirow{3}{*}{2000} & \multirow{3}{*}{2125} & 1 & 0 & 2000 & 0 & 1 & 0 & 0 & 0 & 0 & 0 & 625 \\
\cline { 3 - 14 } & 2 & 625 & 0 & 1500 & 2 & 0 & 0 & 650 & 725 & 250 & 375 \\
\cline { 3 - 13 } & - & - & - & - & 3 & 450 & 500 & 0 & 0 & 550 & 0 \\
\hline
\end{tabular}

Appendix C

Updated plan

The updated plan is generated if there are any changes in data. For experimentation purposes, we analysed the results for 50 test problems that were generated randomly by varying $\pm 20 \%$ of the demand and cost data from the value of ideal plan. The test problems were solved using LINGO to obtain an updated plan in a finite planning horizon of three periods, their total cost patterns are presented in Figure C1.

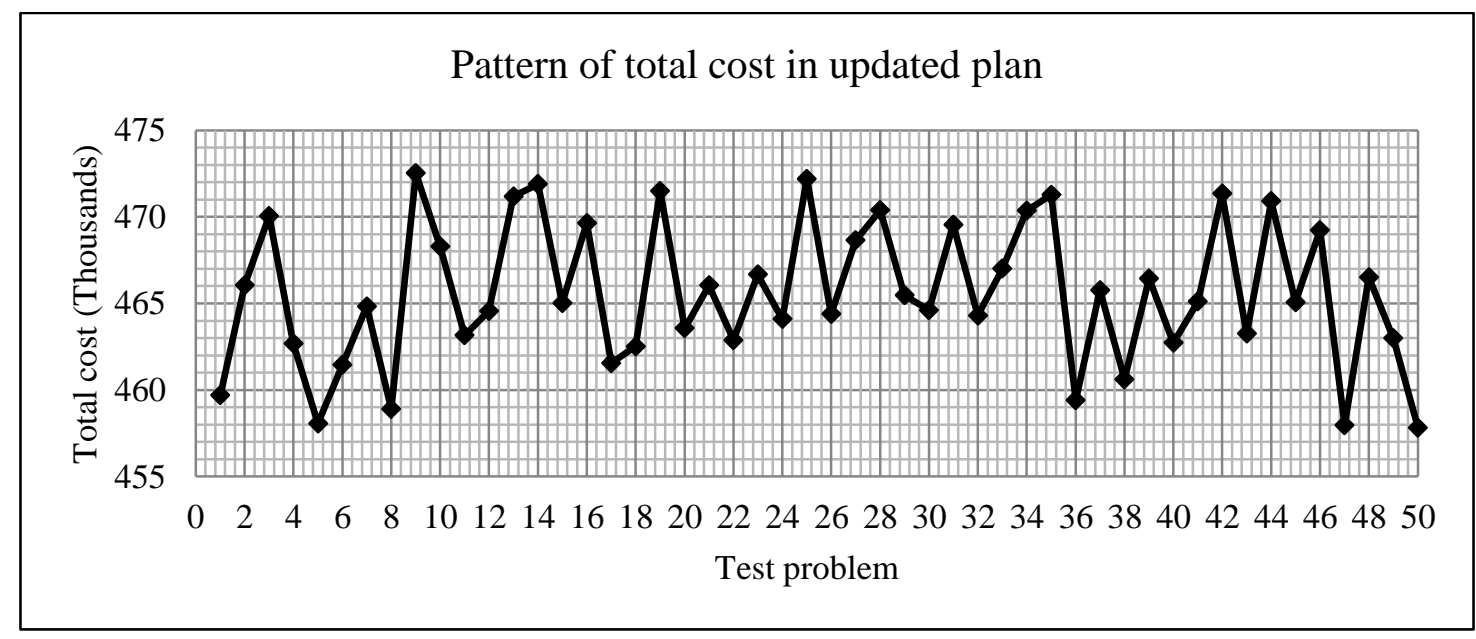

Figure $\mathrm{C} 1$ : Total cost of random experimentation for updated plan 


\section{Appendix D}

\section{Developed pertinent rules for FIS tool}

1. If (Fluctuation is $\mathrm{NH}$ ) then (FIS Value is $\mathrm{NH}$ )

2. If (Fluctuation is NM) then (FIS Value is NM)

3. If (Fluctuation is NL) then (FIS Value is NL)

4. If (Fluctuation is PL) then (FIS Value is PL)

5. If (Fluctuation is PM) then (FIS Value is PM)

6. If (Fluctuation is $\mathrm{PH}$ ) then (FIS Value is $\mathrm{PH}$ )

7. If (Unexpected incident is L) then (FIS Value is PL)

8. If (Unexpected incident M) then (FIS Value is PM)

9. If (Unexpected incident is $\mathrm{H}$ ) then (FIS Value is $\mathrm{PH}$ )

10. If (Natural incident is $\mathrm{NH}$ ) then (FIS Value is $\mathrm{NH}$ )

11. If (Natural incident is NM) then (FIS Value is NM)

12. If (Natural incident is NL) then (FIS Value is NL)

13. If (Natural incident is PL) then (FIS Value is PL)

14. If (Natural incident is PM) then (FIS Value is PM)

15. If (Natural incident is $\mathrm{PH}$ ) then (FIS Value is $\mathrm{PH}$ )

16. If (Fluctuation is $\mathrm{NH}$ ) and (Natural incident is $\mathrm{NH}$ ) then (FIS Value is $\mathrm{NH}$ )

17. If (Fluctuation is $\mathrm{PH}$ ) and (Unexpected incident is $\mathrm{H}$ ) and (Natural incident is $\mathrm{PH}$ ) then (FIS Value is PH) 